\title{
Remote sensing of a NTC radio source from a Cluster tilted spacecraft pair
}

\author{
P. M. E. Décréau ${ }^{1}$, S. Kougblénou*, G. Lointier ${ }^{*}$, J.-L. Rauch ${ }^{1}$, J.-G. Trotignono ${ }^{1}$, X. Vallières ${ }^{1}$, P. Canu ${ }^{2}$, \\ S. Rochel Grimald ${ }^{3}$, F. El-Lemdani $\mathrm{Mazouz}^{4}$, and F. Darrouzet ${ }^{5}$ \\ ${ }^{1}$ Laboratoire de Physique et Chimie de l'Environnement et de l'Espace (LPC2E), UMR7328, Orléans, France \\ ${ }^{2}$ Laboratoire de Physique des Plasmas (LPP), Ecole Polytechnique, Palaiseau, France \\ ${ }^{3}$ Office National d'Études et de Recherches Aérospatiales (ONERA), The French Aerospace Laboratory, Toulouse, France \\ ${ }^{4}$ Laboratoire ATmosphère, Milieux, Observations Spatiales (LATMOS), Guyancourt, France \\ ${ }^{5}$ Belgian Institute for Space Aeronomy (IASB-BIRA), Brussels, Belgium \\ *formerly at: Laboratoire de Physique et Chimie de l'Environnement et de l'Espace (LPC2E), UMR7328, Orléans, France
}

Correspondence to: P. M. E. Décréau (pierrette.decreau@cnrs-orleans.fr)

Received: 15 January 2013 - Revised: 16 May 2013 - Accepted: 24 June 2013 - Published: 26 November 2013

\begin{abstract}
The Cluster mission operated a "tilt campaign" during the month of May 2008. Two of the four identical Cluster spacecraft were placed at a close distance $(\sim 50 \mathrm{~km})$ from each other and the spin axis of one of the spacecraft pair was tilted by an angle of $\sim 46^{\circ}$. This gave the opportunity, for the first time in space, to measure global characteristics of AC electric field, at the sensitivity available with long boom (88 $\mathrm{m}$ ) antennas, simultaneously from the specific configuration of the tilted pair of satellites and from the available base of three satellites placed at a large characteristic separation ( $\sim 1 R_{\mathrm{E}}$ ). This paper describes how global characteristics of radio waves, in this case the configuration of the electric field polarization ellipse in 3-D-space, are identified from in situ measurements of spin modulation features by the tilted pair, validating a novel experimental concept. In the event selected for analysis, non-thermal continuum (NTC) waves in the 15$25 \mathrm{kHz}$ frequency range are observed from the Cluster constellation placed above the polar cap. The observed intensity variations with spin angle are those of plane waves, with an electric field polarization close to circular, at an ellipticity ratio $e=0.87$. We derive the source position in $3-\mathrm{D}$ by two different methods. The first one uses ray path orientation (measured by the tilted pair) combined with spectral signature of magnetic field magnitude at source. The second one is obtained via triangulation from the three spacecraft baseline, using estimation of directivity angles under assumption of circular polarization. The two results are not compatible, placing sources widely apart. We present a general
\end{abstract}

study of the level of systematic errors due to the assumption of circular polarization, linked to the second approach, and show how this approach can lead to poor triangulation and wrong source positioning. The estimation derived from the first method places the NTC source region in the dawn sector, at a large $L$ value $(L \sim 10)$ and a medium geomagnetic latitude $\left(35^{\circ} \mathrm{S}\right)$. We discuss these untypical results within the frame of the geophysical conditions prevailing that day, i.e. a particularly quiet long time interval, followed by a short increase of magnetic activity.

Keywords. Magnetospheric physics (instruments and techniques; plasmasphere)

\section{Introduction}

The Earth produces a variety of radio waves, which have been observed from outer space for a few decades. The three main types of terrestrial radio waves are (i) the terrestrial kilometric radiation (Gurnett, 1974), later on more commonly referred to as the auroral kilometric radiation (AKR); (ii) the non-thermal continuum or NTC (Gurnett, 1975); and (iii) the $2 F_{\mathrm{p}}$ circum terrestrial radiation (Hoang et al., 1981). These three radiations are produced via three different generation mechanisms, from three different regions of the magnetosphere, at three different characteristic frequencies of the source region, respectively the gyrofrequency $F_{\text {ce }}$, the plasma frequency $F_{\mathrm{p}}$, and a multiple of the plasma frequency, 
$2 F_{\mathrm{p}}$. Subsequent observations revealed that all magnetized planets are radio sources (see review by Zarka and Kurth, 2005). Radio waves can travel far from their source region, carrying spectral signatures of the local conditions where they are generated. They thus form a precious tool for remote sensing of the active regions of the magnetosphere, where sources are located. A first target of remote sensing analysis is to determine the position of the sources. Since these active regions are generally located near boundaries, hence restricted to small volumes, a first approach toward localization is to follow the ray path of the radio wave from the point where it is observed. This needs radio wave direction finding at the observation point. The purpose of this paper is to explore a novel instrumental technique for direction finding, to describe how we applied the technique to a chosen case event, and to discuss what has been learned from the exercise.

Radio wave direction finding using the spin modulation of measured electric field intensity by a single receiving antenna has been used for many years (Kurth et al., 1975, 1981; Gurnett, 1975; Etcheto et al., 1982; Gough, 1982; Steinberg et al., 1989; Morgan and Gurnett, 1991; Kasaba et al., 1998; Nagano et al., 2003; Décréau et al., 2004; Grimald et al., 2007). This tool, sometimes referred to as "spin-null method", uses a rotating directive antenna (Calvert, 1999). It yields ray path orientation in the spin plane of the satellite, indicated by the position of a rotating antenna at minimal received intensity, preferably near null for a better qualification of the method. It is based on simple assumptions about wave properties, either that the radio source is un-polarized, or, more currently, that the measured electric field vector is that of a monochromatic plane wave, circularly polarized, travelling in free space. Within that frame, the unit vector $\boldsymbol{k}$ normal to the electric field plane is the wave vector, aligned with the ray path; the electric field projected in the spin plane describes an ellipse whose small axis is aligned with the projection onto this same plane of the $\boldsymbol{k}$ vector.

Although it was recognized early on that erroneous source direction could be produced by polarization effects (Jones, 1982), ray path orientations of NTC waves have been widely derived using the "spin-null method" using the circular polarization assumption. The experimental evidence of quasicircular polarization has been obtained from NTC observations by the DE-1 satellite (Gurnett et al., 1988). Furthermore, first determinations of NTC ray path orientation derived under such a model did provide encouraging results pointing, for instance, toward realistic source positions, like equatorial plasmapause, or magnetopause (Gurnett, 1975; Etcheto et al., 1982; Gough, 1982). One important application of this technique is its capacity, under favourable configurations of the orbit and source, to derive the source position by triangulation (Grimald et al., 2009). Furthermore, the modulation factor (defined when comparing levels of minimal to maximal intensity within a spin), has been used to derive the size of sources (Steinberg et al., 1989), or to estimate the angle between ray path and spin plane, hence ray path direction in 3-D (Grimald et al., 2011).

The "spin-null method" made use of an opportunity offered by scientific satellites equipped with spinning long booms measuring electric field, but could not satisfactorily resolve the localization in 3-D of a radio source of unknown polarization, a general objective of radio astronomy based on satellite observations. The ideal equipment is a set of three antennas of same characteristics, deployed along three orthogonal directions, connected to electronic devices measuring phase differences between any two of the three signals received. This would yield a complete characterization of the wave electric field, including its sense of polarization. The main difficulty in space is that long antennas (of good sensitivity) deployed via spinning are confined into the spin plane, and that rigid antennas cannot be deployed beyond a few metres, i.e. in a volume where sheath effects can be problematic. In practice, two types of equipment allowing 3-D measurements of electric field have been flown and tested in space. One type is a rigid short antenna, placed along the spin axis of a spacecraft equipped with one or two long boom spinning antennas. The other type is a set of three rigid antennas, placed either on a spinning spacecraft or on a 3-axes stabilized spacecraft. How to make the best use of such devices in order to do reliable direction finding at a good resolution is a difficult task. Specific studies have been devoted to examine this question and to design antenna systems (Lecacheux, 1978; Fainberg et al., 1985; Ladreiter et al., 1995; Cecconi and Zarka, 2005). Both types of equipment have demonstrated their usefulness. The mixed device (spinning antennas associated to a rigid one) has been used on DE-1 to derive the polarization of AKR (Shawhan and Gurnett, 1982), and on WIND to derive properties of the $2 F_{\mathrm{p}}$ radio emission (Reiner et al., 1996). Polarization properties of AKR have also been obtained by sets of three rigid antennas. Hanasz et al. (2000) derived dynamic spectra of Stokes parameters from measurements aboard the spinning Interball-2 spacecraft, and Lamy et al. (2010) used equipment placed on the 3-axes stabilized Cassini orbiter to disentangle left-handed from right-handed waves.

The Cluster constellation offered another approach, where two sets of long boom spinning antennas, placed on board two different satellites, could be used for electric field measurements in 3-D. Cluster, the first space mission designed to study small-scale space plasma structures in three dimensions (Escoubet et al., 1997), is a constellation of four identical satellites (C1, C2, C3, C4). The Cluster satellites spin at a period of about $4 \mathrm{~s}$, with, under nominal conditions, all spin axes parallel to each other, oriented normal to the ecliptic plane. The satellites were placed mostly in a regular tetrahedron configuration during the first years of the mission. Later on, they were placed on several occasions in a multi-scale configuration, where three satellites $(\mathrm{C} 1, \mathrm{C} 2$ and $\mathrm{C} 3)$ form a large triangle, and the fourth one (C4) is placed at a short distance from C3. The two satellites placed close by, C3 and C4, 
share the same orbit and their separation distance is easily adjustable. During one such configuration in May 2008 (socalled "tilt campaign"), the spin axis of $\mathrm{C} 3$ was tilted at an angle of about $45^{\circ}$. For the first time, electric field measurements at the sensitivity available with long boom antennas were accessible in 3-D, as the two spacecraft placed within $\sim 50 \mathrm{~km}$ distance of each other could be considered as a single observatory. This context opened the path to a new way of measuring electric field polarization properties. This could be used not only to estimate the ray path orientation in 3-D but also to study the wave mode of propagating emissions (see Carozzi et al., 2000), noting that only the global shape of the polarization ellipse could be identified by the existing instrumentation, since the phase difference between signals measured on board the two spacecraft is not available.

We concentrate here on radio wave direction finding via data obtained by the WHISPER (Waves of HIgh frequency and Sounder for Probing of Electron density by Relaxation) instrument (Décréau et al., 1997). WHISPER data were available during most of the one month tilt campaign, while measurements from the WBD (WideBand Data) instrument (Gurnett et al., 1997), covering a similar frequency range with better time and frequency resolutions, were by necessity restricted to narrow time windows during the campaign. We will show below that the time and frequency resolution available on WHISPER (respectively $1.7 \mathrm{~s}$ and $0.64 \mathrm{kHz}$ ) are sufficient to measure the characteristics of spin modulation and to resolve details of the spectral signatures (electron gyro-frequency at the source), allowing remote sensing of the source region. How to interpret data obtained in the tilted pair configuration by the coupled WHISPER instruments was a challenge. Indeed, measurement performances explored before the campaign with synthetic signal tests and realistic operation schemes have been shown to vary significantly according to the actual orientation of the polarization ellipse with respect to the tilted constellation geometry. Hence, some geometries could be unfavourable, leading to large uncertainties in derived ellipse characteristics. This difficulty is actually inherent to any direction finding system (see error analysis in Cecconi and Zarka, 2005). Another difficulty inherent in the use of real data is that measured intensities result in general from a superposition of wavelets, stemming from micro sources distributed over a source region of finite size. The measured modulation factor is in such a case smaller than it would be when observing a pure wave. As discussed in Gurnett (1975), its value could be used to roughly estimate the angular size of sources, provided that ray paths lie in the spin plane. In this study, and it is important to stress this point, we ignore the effect of the angular size of the source as observed from Cluster.

The prime purpose of this paper is to present the results obtained from a custom-built software tool, which retrieves the characteristics of the ellipse described by the electric field of a single plane wave, without any assumption about orientation of ray path with respect to spin plane. We call this tool the Dual Spin Modulation, or DSM tool. We apply the DSM tool to NTC radiation measured during the tilt campaign. Studies of NTC waves by the multi-point Cluster constellation has allowed to better understand and decipher the observed characteristics of spectral signatures of this radiation, and to translate them into genuine characteristics of the source region (Masson et al., 2009; Grimald et al., 2009, 2011), thus forming a good background for the analysis of NTC events using the tilted pair. The chosen event deals with NTC data measured during a time interval of about one hour, when all four spacecraft travel above the southern polar cap in a region of free propagation for the considered frequencies. This allows to search for the source position by simple ray tracing (the radiation travelling along a straight line).

We present in Sect. 2 the DSM tool. Section 3 shows the application of this tool to a WHISPER elementary data set (covering the short time interval necessary to derive modulation characteristics). The same section discusses practical ways of appreciating the validity of the circular polarization assumption. Section 4 presents the selected event, the DSM analysis applied to a larger time interval, leading to an averaged ray path direction. Section 5 presents the search for source position and discusses the results achieved. We compare the results obtained with the more partial information that would have been available in the absence of the tilted spacecraft. Finally, we draw general conclusions in Sect. 6 .

\section{The dual spin modulation tool}

\subsection{Measured quantities}

One characteristic feature of observed NTC radio waves is their duration of typically several minutes, contrasting with the sporadic nature of AKR radio waves. Even if it does not imply coherence of NTC wave trains during such long durations, direction finding studies rely on wave properties (beam position and field intensity) being quasi-stationary during time intervals of the order of one minute. When dealing with wave measurements obtained from two different spacecraft, we assume in addition that both platforms are located inside the same beam and that the distances from each satellite to the source are close enough for field intensities to be equal.

The measuring system at our disposal is made of two long boom receiving antennas on the $\mathrm{C} 3$ and $\mathrm{C} 4$ satellites that are placed at an inter-satellite distance of about $50 \mathrm{~km}$ during the tilt campaign. The two antennas are spinning, independently from each other, at periods slightly different, of about $4 \mathrm{~s}$. They are connected respectively to the $\mathrm{C} 3$ and $\mathrm{C} 4$ WHISPER instruments, which perform onboard frequency analysis of the electric field signal measured in the $2-80 \mathrm{kHz}$ frequency band (Décréau et al., 2001). In order to do so, each antenna samples the electric signal during time acquisition intervals of short duration $(3.1 \mathrm{~ms})$, during which the receiving antenna can be assumed to keep the same attitude 

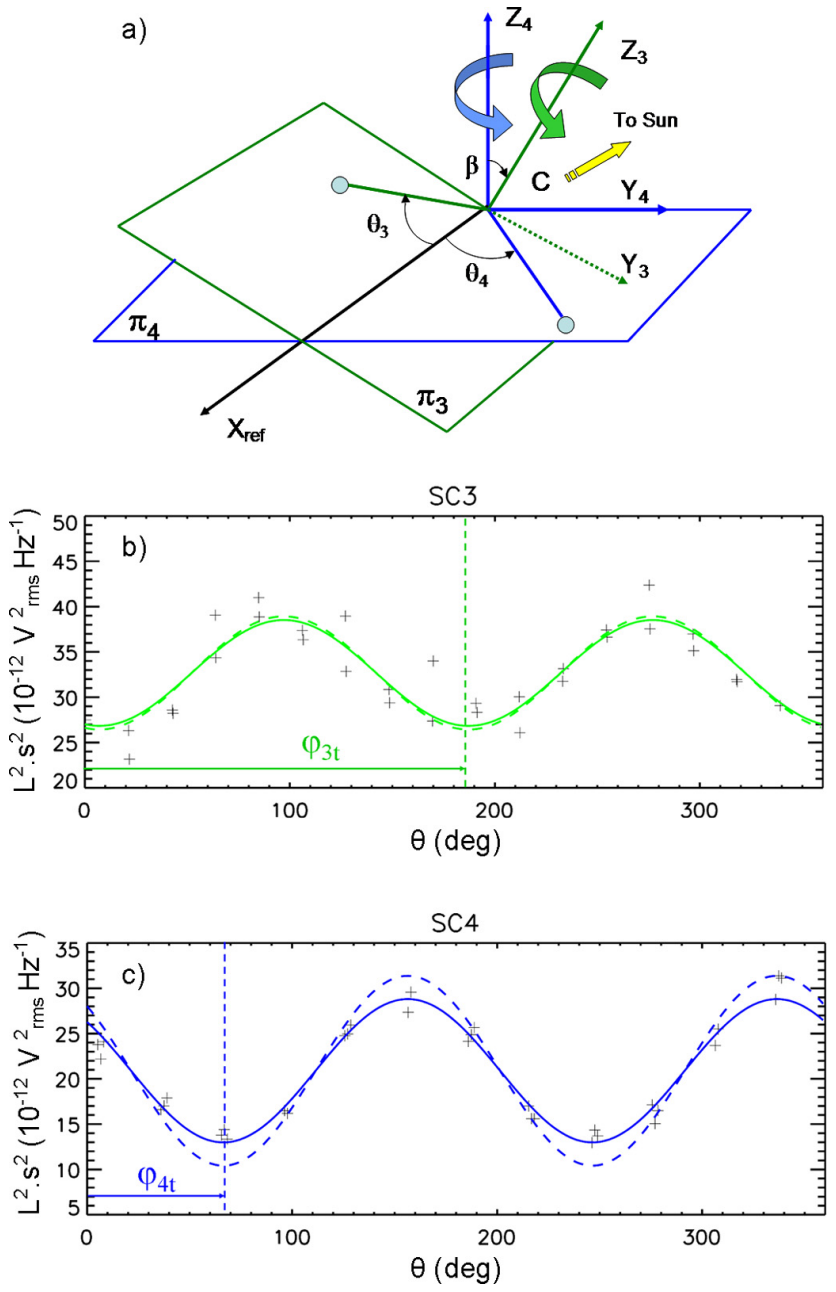

Fig. 1. (a) Configuration of spin axes $\left(Z_{3}\right.$ and $Z_{4}$, making an angle $\beta$ ) and spin planes $\left(\pi_{3}\right.$ and $\left.\pi_{4}\right)$ of the $C 3-C 4$ pair. Curved arrows indicate the sense of rotation (with time) of each receiving antenna, sketched as half a dipole. The $\left[S_{\mathrm{C} 4}\right]$ and $\left[S_{\mathrm{C} 3}\right]$ coordinate systems are defined by the axes $Z_{4}$ (respectively $Z_{3}$ ), $X_{\text {ref }}$ (common axis along intersection of spin planes) and $Y_{4}$ (respectively $Y_{3}$ ). The antenna attitude angles, $\theta_{4}$ on $\mathrm{C} 4$ and $\theta_{3}$ on $\mathrm{C} 3$, are expressed from the common origin $X_{\text {ref }}$ to the unit vector associated to the antenna. (b) and (c) Electric field spectral power densities in the $15-25 \mathrm{kHz}$ band and integrated over the antenna length $L$, sampled over a one minute time interval (18 May 2008, 12:20 to $12: 21$ UTC) at a $1.7 \mathrm{~s}$ recurrence, are plotted (grey crosses) versus attitudes $\Theta$ at sampling times, respectively for C3 (top) and C4 (bottom). Solid lines display a fit to the measured quantities according to a modelled spin variation. Dashed lines display the derived instantaneous values of the modelled electric field (see text). Quantities $\phi_{3 \mathrm{t}}$ and $\phi_{4 \mathrm{t}}$ are best estimates of attitudes at minimal intensity.

$\theta$. The acquisition intervals are placed $13.3 \mathrm{~ms}$ apart. Successive electric field magnitude frequency spectra, calculated by FFT (fast Fourier transform) every $13.3 \mathrm{~ms}$, are accumulated on board by the DWP (Digital Wave Processing) instrument, bin to bin, to form a single "accumulated" spectrum (Woolliscroft et al., 1997; Trotignon et al., 2010). Due to different operating capabilities of WHISPER instruments on board C3 and C4 (the latter having lost a memory word in year 2003), "accumulated" spectra are available more frequently on C3 (32 individual spectra accumulated every $0.426 \mathrm{~s}$ ) than on C4 (64 individual spectra accumulated every $0.852 \mathrm{~s}$ ). The telemetry bit rate allocated to WHISPER allows transmitting to ground every second one of the accumulated spectra for $\mathrm{C} 4$, and one out of four accumulated spectra for $\mathrm{C} 3$. As a result, the transmission recurrence, $1.7 \mathrm{~s}$, is the same on both satellites. The difference between measurement times and half-spin periods ( $\sim 2 \mathrm{~s}$ on each satellite) is such that the antenna samples varying attitude positions as time progresses, thus exploring how the electric field magnitude is modulated with respect to attitude.

Figure 1 presents WHISPER measurements obtained during a one minute time interval. Assuming that the observed NTC waves are of the same nature as those analysed via the "spin-null method" in the past, i.e. plane waves and quasicircular polarization, one expects that each one of the two satellites will present a spin modulation of the measured electric field. This is actually the case, as shown in the figure. In order to compare both spin modulation characteristics, we define suitable coordinate systems, sketched in Fig. 1a. Despun coordinate systems of $\mathrm{C} 3$ and $\mathrm{C} 4$ are translated such that $\mathrm{C} 3$ and $\mathrm{C} 4$ are superposed in $\mathrm{C}$, at the intersection of translated spin axes $Z_{3}$ and $Z_{4}$. We actually assume that the global properties of the measured NTC field are unchanged from one spacecraft position to the other, such that the two satellites form a single measuring system, equipped with two identical long antennas, sweeping two spin planes at an angle to each other. The orientation of unit vector along $X_{\text {ref }}$ is chosen to be given by the cross product of $\boldsymbol{K}_{3}$ and $\boldsymbol{K}_{4}$, unit vectors respectively along $Z_{3}$ and $Z_{4}$ axis. This results in a direction $X_{\text {ref }}$ roughly opposite to the Sun. The axis $Y_{4}$ completes a coordinate system $\left[X_{\text {ref }}, Y_{4}, Z_{4}\right]$ attached to $\mathrm{C} 4$. Let us call $\left[S_{\mathrm{C} 4}\right]$ this coordinate system. Similarly, we define a coordinate system $\left[S_{\mathrm{C} 3}\right]$ attached to $\mathrm{C} 3$, based on axes $X_{\text {ref }}, Z_{3}$ and a third axis $Y_{3}$ in $\pi_{3}$, spin plane of C3. [ $S_{\mathrm{C} 3}$ ] can be derived from $\left[S_{\mathrm{C} 4}\right]$ by a rotation around $X_{\text {ref }}$ of angle $\beta$, equal to $46.1^{\circ}$, between spin axes $Z_{4}$ and $Z_{3}$. Attitudes $\theta_{4}$ and $\theta_{3}$ are expressed as the angle $\theta$ between each spinning (double sphere) receiving antenna and the common direction $X_{\text {ref }}$, defined by the intersection of the two spin planes.

Panels (b) and (c) in Fig. 1 show accumulated electric field spectral power densities (crosses) measured within the same 60 s time interval, starting at 12:20 UTC on 18 May 2008, plotted as a function of the attitude of the respective antennas at central times of accumulation intervals. We notice that the total number of samples acquired during the same UTC interval is slightly different on C3 (33 samples) and on C4 (34 samples) due to time sequences which are not synchronized better than to a one second boundary from one satellite to the other (onboard clocks tuned to UTC). Moreover, attitudes sampled on each spacecraft differ since the spin periods 
are slightly different. As expected, the measured signals are clearly modulated with the respective attitude angles $\theta$. For each satellite, electric field spectral power densities follow variation $s^{2}(\theta)$ (solid lines in Fig. 1b and c) expressed in Eq. (1):

$s_{\mathrm{m}}^{2}=E_{0}^{2}\left[1-\alpha_{0} \cos 2\left(\theta-\phi_{\mathrm{t}}\right)\right]$.

Three adjustable parameters, $E_{0}, \alpha_{0}$, and $\phi_{\mathrm{t}}$, have been determined for each spacecraft by minimizing, in the sense of least square approximation, the distance between the measurements and electric field power densities of Eq. (1). The quantity $E_{0}^{2}$ represents the average value of modulated signal, $E_{0}^{2}=\frac{s_{\mathrm{m} \_ \text {max }}^{2}+s_{\mathrm{m} \_ \text {min }}^{2}}{2}$, where $s_{\mathrm{m} \_ \text {max }}^{2}$ and $s_{\mathrm{m} \_ \text {min }}^{2}$ are extrema of $s_{\mathrm{m}}^{2}$. The quantity $\alpha_{0}$ represents the modulation factor of the measured signal, $\alpha_{0}=\frac{s_{\mathrm{m} \_ \text {max }}^{2}-s_{\mathrm{m} \text { min }}^{2}}{s_{\mathrm{m} \_ \text {max }}^{2}+s_{\mathrm{m} \_ \text {min }}^{2}}$ (as in Steinberg et al., 1989, or in Grimald et al., 2011), and the quantity $\phi_{\mathrm{t}}$ is the antenna attitude at minimal signal. Note that in Eq. (1), $s_{\mathrm{m}}^{2}$ and $E_{0}^{2}$ stand for electric field spectral power densities (expressed in units of $V_{\mathrm{rms}}^{2} \mathrm{~m}^{-2} \mathrm{~Hz}^{-1}$ ), while Fig. $1 \mathrm{~b}$ and c display the spectral power density of electric field integrated along the effective length of the antenna, a quantity expressed in units of $V_{\mathrm{rms}}^{2} \mathrm{~Hz}^{-1}$. The latter quantity, closer to the raw measurement, is directly proportional to the former, according to a coefficient identical on both spacecraft, since their respective receiving antenna have the same physical length and provided that the plasma regime is similar enough on each platform for effective lengths to be equal to each other (Béghin et al., 2005).

An important remark concerns the estimation of the modulation factor. As stated above, the WHISPER instrument reports the electric field frequency spectra accumulated within time intervals, $426 \mathrm{~ms}$ for $\mathrm{C} 3$ and $852 \mathrm{~ms}$ for $\mathrm{C} 4$, during which the antenna attitudes varies significantly. The accumulated intensities are squeezed toward the average intensity $E_{0}^{2}$ and the measured modulation factor $\alpha_{0}$ underestimates the modulation factor $\alpha$, which would reflect instantaneous spectral magnitudes initially measured. Post hoc data analysis takes this problem into account and calculates the true modulation factor $\alpha$ by introducing a correction factor. The correction factor has been calculated by applying all actual onboard operations, mainly onboard data accumulation, to a synthetic signal figuring the instantaneous spectral magnitudes measured by the receiving antenna. The calculated correction factor depends only on the number of accumulated spectra. Finally, the quantities measured are three adjustable parameters, $E_{0}, \alpha$, and $\phi_{\mathrm{t}}$, responding to Eq. (2), where $s^{2}$ represent the instantaneous spectral power (dashed lines in Fig. $1 \mathrm{~b}$ and c):

$s^{2}=E_{0}^{2}\left[1-\alpha \cos 2\left(\theta-\phi_{t}\right)\right]$.

According to Eq. (2), the instantaneous spectral power oscillates between extrema $s_{\max }^{2}=E_{0}^{2}(1+\alpha)$ and $s_{\min }^{2}=E_{0}^{2}$ $(1-\alpha)$. We shall see in the next subsection that modulation factors play a crucial role in the algorithm, which allows retrieving ellipse characteristics.

In summary, six spin modulation parameters are derived from raw measurements, the set $E_{03}, \alpha_{3}$, and $\phi_{3 t}$ and the set $E_{04}, \alpha_{4}$, and $\phi_{4 \mathrm{t}}$ calculated from WHISPER spectra measured respectively on C3 and C4. Spectral power density variations with attitude angle differ significantly when measured on one spacecraft or on the other. We note, however, that wave powers measured at attitude (or phase) zero by $\mathrm{C} 3$ and $\mathrm{C} 4$ are close to each other, equal to 27 and 28, respectively, in the chosen unit. This is not by accident, but because at phase zero respective receiving antenna are aligned with each other, along the common direction defined by the intersection of the two spin planes. In practice, only five independent spin modulation parameters are measured by the tilted pair when based on spectra acquired during the same time interval and over the same frequency band.

\subsection{Direct problem: measured quantities as a function of ellipse characteristics}

The electric field $E(f)$ of a sinusoidal wave at frequency $f$ traces out an ellipse in the plane of electric polarization. The polarized electric field $\boldsymbol{E}$ can be represented as the superposition of two linearly polarized waves at right angles, of respective magnitudes $a$ and $b$, varying with time in quadrature according to the following equation:

$E(t)=a \cos \omega_{0} t \boldsymbol{u} \pm b \sin \omega_{0} t \boldsymbol{v}$,

where $\omega_{0}$ is the angular pulsation at frequency $f, a$ and $b$ are lengths of the semi major and semi minor axes of the polarization ellipse, $\boldsymbol{u}$ and $\boldsymbol{v}$ the associated unit vectors, and $\boldsymbol{u}, \boldsymbol{v}$, $\boldsymbol{k}$ are forming an orthogonal base (see, for instance, Calvert, 1999). The aim of the analysis is to measure the quantities $\boldsymbol{u}$, $\boldsymbol{v}, a$ and $b$, which fully describe a given ellipse configuration (see sketch in Fig. 3a). As only wave intensity is measurable, the sense of polarization (left for a plus sign in Eq. (3), right for a minus sign) is unknown. Moreover, the direction of each vector is known with an ambiguity of $\pi$, such that the order of unity vectors $\boldsymbol{u}, \boldsymbol{v}, \boldsymbol{k}$ with respect to a direct base is unspecified.

We choose the coordinate system $\left[S_{\mathrm{C} 4}\right]$ defined above as the reference system where we express the vector quantities used to describe the polarization ellipse. The orientation of unit vector $\boldsymbol{u}$ is defined by two angles in a spherical coordinate system, the zenith angle $\sigma_{a}$ and the azimuth $\mu_{a}$, sketched in Fig. 2a. Similarly, the orientation of unit vector $v$ is defined by the zenith angle $\sigma_{b}$ and the azimuth $\mu_{b}$ (not shown in the figure). Taking account of the property of vector $\boldsymbol{v}$ to be normal to vector $\boldsymbol{u}$, only three independent parameters are required to fully describe the orientation of $\boldsymbol{u}$ and $\boldsymbol{v}$. Added to the lengths of ellipse axes $a$ and $b$, this amounts to five independent parameters, which the analysis via the DSM tool aims to calculate from the set of five independent 
a)

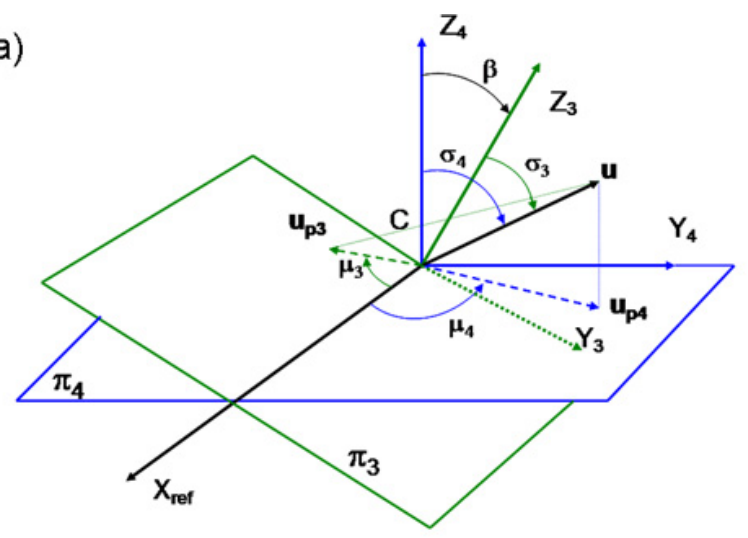

b)

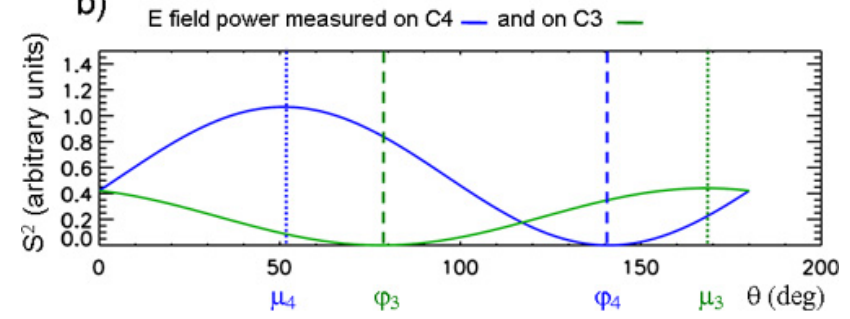

c)

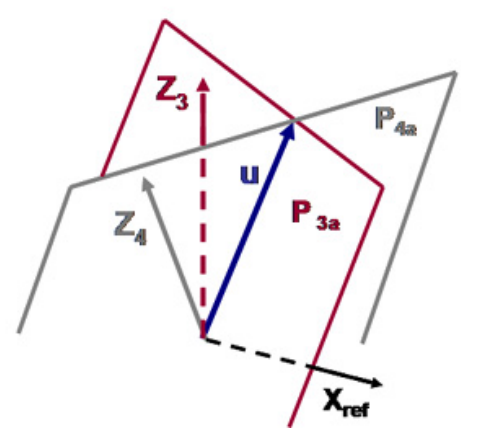

Fig. 2. Case of linear polarization. (a) Sketch of unit vector $\boldsymbol{u}$ of an electric field vector of magnitude a in the tilted pair system. The orientation of $\boldsymbol{u}$ is defined by the two angles, $\sigma_{4}$ and $\mu_{4}$ in spherical coordinates of the $\left[S_{\mathrm{C} 4}\right]$ system, alternatively $\sigma_{3}$ and $\mu_{3}$ in the $\left[S_{\mathrm{C} 3}\right]$ coordinate system. Vectors $\boldsymbol{u}_{\mathrm{p} 4}$ and $\boldsymbol{u}_{\mathrm{p} 3}$ are projections of vector $\boldsymbol{u}$ onto spin planes. (b) Electric field power variations plotted versus antenna attitudes angles in a chosen case (see text). They reach a maximum when the antenna is aligned with $\boldsymbol{u}_{\mathrm{p} 4}$ and $\boldsymbol{u}_{\mathrm{p} 3}$ (attitude angles $\mu_{4}$ and $\mu_{3}$ ), and is null when the antenna is at right angles to the projections (angles $\varphi_{4}$ and $\varphi_{3}$ ). (c) The two meridian planes $P_{4 a}$ and $P_{3 a}$, attached respectively to $\left[S_{\mathrm{C} 4}\right]$ and $\left[S_{\mathrm{C} 3}\right]$ coordinate systems, intersecting along vector $\boldsymbol{u}$.

measured quantities described above (Sect. 2.1). The direct problem, i.e. the relationship between the set of input quantities $\sigma_{a}, \mu_{a}, \sigma_{b}, \mu_{b}, a, b$, and the set of output quantities $E_{04}$, $\alpha_{4}, \phi_{4 \mathrm{t}}, E_{03}, \alpha_{3}, \phi_{3 \mathrm{t}}$, is obtained as follows.

The wave component linearly polarized along vector $\boldsymbol{u}$ of pulsation $\omega_{0}$ produces an electric field varying, when projected onto the spin plane of satellite $\mathrm{C} 4$, as $\boldsymbol{E}_{\mathrm{p}}(t)=$

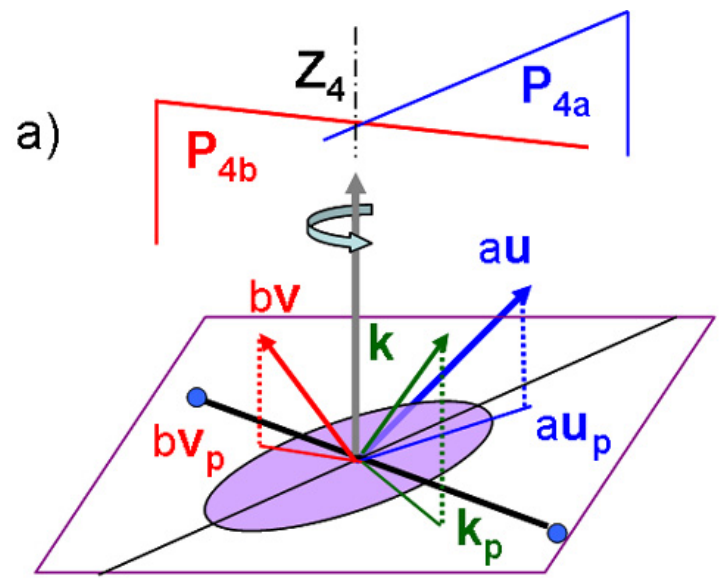

spin plane

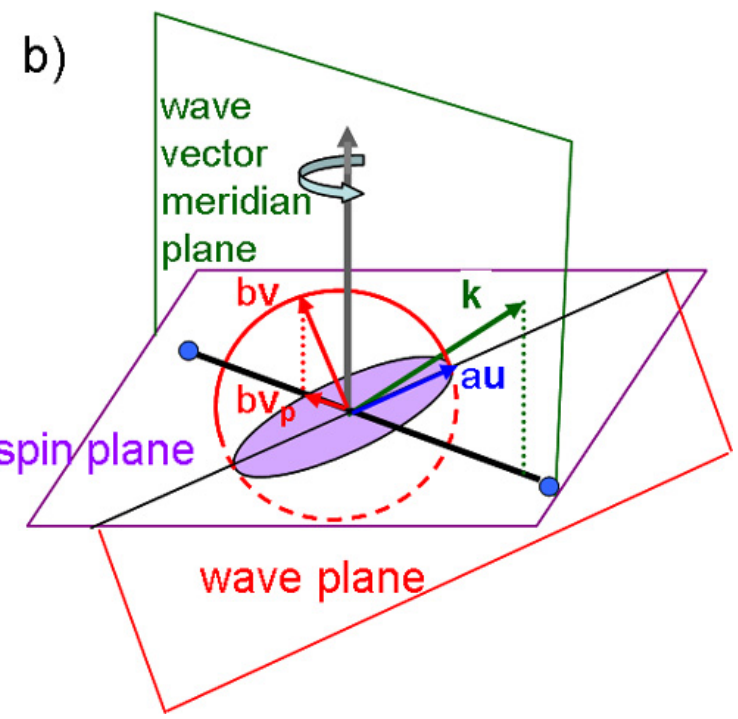

Fig. 3. Case of elliptic polarization. (a) Linear components $a \boldsymbol{u}$ and $b \boldsymbol{v}$ of an electric field elliptically polarized. Vectors $\boldsymbol{u}$ and $\boldsymbol{v}$ of the wave plane and its normal $\boldsymbol{k}$ are shown in $\left[S_{\mathrm{C} 4}\right]$ coordinate system. Meridian planes $P_{4 a}$ and $P_{4 b}$ contain $Z_{4}$ axis and, respectively, $\boldsymbol{u}$ and $\boldsymbol{v}$ vectors. Projections $\boldsymbol{u}_{\mathrm{p}}, \boldsymbol{v}_{\mathrm{p}}$ and $\boldsymbol{k}_{\mathrm{p}}$ of $\boldsymbol{u}, \boldsymbol{v}$ and $\boldsymbol{k}$ onto $\mathrm{C} 4$ spin plane are shown, as well as the electric field ellipse projected onto the same plane (purple area). (b) Specific case of circular polarization where principal axis positions have been chosen such that $\boldsymbol{u}$ vector was in the spin plane. Projections $\boldsymbol{u}_{\mathrm{p}}\left(\boldsymbol{u}_{\mathrm{p}}=\boldsymbol{u}\right)$ and $\boldsymbol{v}_{\mathrm{p}}$ are at a right angle to each other. The meridian plane (drawn in green) formed by the spin axis and $\boldsymbol{v}_{\mathrm{p}}$ contains the wave vector $\boldsymbol{k}$.

$a \cos \omega_{0} t \boldsymbol{u}_{\mathrm{p}}$. The antenna captures the electric field vector projected along its direction defined by the unit vector $\boldsymbol{U}$, placed at attitude angle $\theta$. The magnitude of the received signal, $A=a \boldsymbol{u}_{\mathrm{p}} \boldsymbol{U}$, is then

$A=a \sin \sigma_{a} \cdot \cos \left(\mu_{a}-\theta\right)$. 
The corresponding average power $s_{a}^{2}=A^{2} / 2$, which is the quantity actually available after onboard analysis, can be expressed, when defining the azimuth of $\boldsymbol{u}$ via $\phi_{a}=\mu_{a} \pm \pi / 2$, as $s_{a}^{2}=\frac{a^{2}}{2} \sin ^{2} \sigma_{a} \sin ^{2}\left(\theta-\phi_{a}\right)$, which leads to

$s_{a}(t)^{2}=m_{a}^{2}\left[1-\cos 2\left(\theta(t)-\phi_{a}\right)\right]$, where $m_{a}^{2}=\frac{1}{4} a^{2} \sin ^{2} \sigma_{a}$.

Figure $2 b$ illustrates the variation of electric field power versus attitude created by a linearly polarized wave component (blue curve), displaying a maximum when the antenna is aligned with $\boldsymbol{u}_{\mathrm{p}}\left(\theta=\mu_{a}\right)$ and a null when the antenna is normal to $\boldsymbol{u}_{\mathrm{p}}\left(\theta=\phi_{a}\right)$. Similarly, the second wave component linearly polarized along vector $\boldsymbol{v}$ (not shown in Fig. 2a) creates the average power:

$s_{b}(t)^{2}=m_{b}^{2}\left[1-\cos 2\left(\theta(t)-\phi_{b}\right)\right]$, where $m_{b}^{2}=\frac{1}{4} b^{2} \sin ^{2} \sigma_{b}$.

The measured signal $s_{t}^{2}$ is the sum of both contributions. Expressed as a function of the attitude $\theta$, which explores the $[0-2 \pi]$ interval during a spin period, it is the sum of two sine functions, of half the spin periodicity, which varies as a sine function of the same period (Fig. A1 in the Appendix), according to the model of Eq. (2):

$s_{\mathrm{t}}^{2}=E_{04}^{2}\left[1-\alpha_{4} \cos 2\left(\theta-\varphi_{4 \mathrm{t}}\right)\right]$.

The identity $s_{a}^{2}+s_{b}^{2} \equiv s_{\mathrm{t}}^{2}$ (developed in the Appendix) yields to the set $E_{04}, \alpha_{4}, \phi_{4 \mathrm{t}}$ via the following relations:

$E_{04}^{2}=\frac{1}{4} a^{2} \sin ^{2} \sigma_{a}+\frac{1}{4} b^{2} \sin ^{2} \sigma_{b}$

$\varphi_{4 \mathrm{t}}=\phi_{a}-\frac{1}{2} \delta, \quad$ with $\delta=\operatorname{atan} \frac{r \sin \zeta}{1+r \cos \zeta}, \quad$ where

$\zeta=2\left(\varphi_{a}-\varphi_{b}\right) \quad$ and $r=\operatorname{Min}\left[\frac{a^{2} \sin ^{2} \sigma_{a}^{2}}{b^{2} \sin ^{2} \sigma_{b}^{2}}, \frac{b^{2} \sin ^{2} \sigma_{b}^{2}}{a^{2} \sin ^{2} \sigma_{a}^{2}}\right]$

$\alpha_{4}=\frac{1+r \cos \zeta}{(1+r) \cos \delta}$

The quantities $E_{03}, \phi_{3 \mathrm{t}}$ and $\alpha_{3}$, measured on $\mathrm{C} 3$, can be expressed by the same equations, as function of the set $\sigma_{a}^{\prime}, \mu_{a}^{\prime}$, $\sigma_{b}^{\prime}, \mu_{b}^{\prime}, a$ and $b$, where $\sigma_{a}^{\prime}, \mu_{a}^{\prime}, \sigma_{b}^{\prime}, \mu_{b}^{\prime}$ are the spherical coordinates of $\boldsymbol{u}$ and $\boldsymbol{v}$ vectors expressed in the coordinate system $\left[S_{\mathrm{C} 3}\right]$ linked to $\mathrm{C} 3$. The quantities $\sigma_{a}^{\prime}, \mu_{a}^{\prime}, \sigma_{b}^{\prime}, \mu_{b}^{\prime}$ are then replaced in the equations by their expression in function of input quantities $\sigma_{a}, \mu_{a}, \sigma_{b}, \mu_{b}$ via a change of coordinate system, from $\left[S_{\mathrm{C} 3}\right]$ to $\left[S_{\mathrm{C} 4}\right]$.

\subsection{Inverse problem: deriving ellipse configuration from measured quantities}

Let us first present the specific case of linear polarization, which illustrates (and inspired) the approach presented for the general case. Under linear polarization, the electric field can be expressed as $E(t)=a \cos \omega_{0} t \boldsymbol{u}$. Projected onto each spin plane, it oscillates along a line aligned with the projections of vector $\boldsymbol{u}$ onto spin planes. Figure 2a shows the configuration of the vector $\boldsymbol{u}$ and of its projections $\boldsymbol{u}_{\mathrm{p} 4}$ and $\boldsymbol{u}_{\mathrm{p} 3}$ onto planes $\pi_{4}$ and $\pi_{3}$, spin planes of $\mathrm{C} 4$ and $\mathrm{C} 3$. The vector $\boldsymbol{u}_{\mathrm{p} 4}$ is placed in the meridian plane $\left(Z_{4}, \boldsymbol{u}\right)$ of the $\left[S_{\mathrm{C} 4}\right]$ coordinate system, $P_{4 a}$, sketched in Fig. 2c. Similarly, the vector $\boldsymbol{u}_{\mathrm{p} 3}$ is placed in the meridian plane $\left(Z_{3}, \boldsymbol{u}\right)$ of the $\left[S_{\mathrm{C} 3}\right]$ coordinate system, $P_{3 a}$, also sketched in Fig. 2c. As C4 receiving antenna rotates around $Z_{4}$, the electric field intensity varies at half $\mathrm{C} 4$ spin period, according to Eq. (4). The intensity variation with attitude is plotted in Fig. $2 \mathrm{~b}$ (blue curve) in the specific case where $\sigma_{4}=47^{\circ}$ and $\mu_{4}=51^{\circ}$. In this case, the coordinates of vector $\boldsymbol{u}$ are $\sigma_{3}=28^{\circ}$ and $\mu_{3}=168^{\circ}$ when expressed in $\left[S_{\mathrm{C} 3}\right]$. The intensity variation with attitude observed on $\mathrm{C} 3$ is plotted in Fig. $2 \mathrm{~b}$ in green. The minimal intensities are equal to zero on both spacecraft, providing a means to identify occurrences of this particular case. The antenna attitudes at maximal intensities on $\mathrm{C} 4$ and on C3 identify the longitudes of the meridian planes $P_{4 a}$ and $P_{3 a}$, respectively in $\left[S_{\mathrm{C} 4}\right]$ and $\left[S_{\mathrm{C} 3}\right]$ systems.

Finally, the orientation of $\boldsymbol{u}$ vector is aligned with the intersection of meridian planes $P_{3 a}$ and $P_{4 a}$ (Fig. 2c). It is calculated as the cross product of their normal vectors, defined respectively by angles $\phi_{4}$ and $\phi_{3}$ in each spin plane. The result is expressed in the chosen common coordinate system, [ $\left.S_{\mathrm{C} 4}\right]$. Having identified the orientation of $\boldsymbol{u}$ vector in 3-D, it is possible to calculate the magnitude $a$ of electric field from characteristic power values $m_{a}^{2}$ measured either on $\mathrm{C} 4$ or on C3 (Eq. 4). Both estimations of $a$ should be equal if the model of a linear electric field, homogeneous over the $\mathrm{C} 3$ to $\mathrm{C} 4$ distance, is valid. This has been verified in case events of linearly polarized whistler waves recorded during the tilt campaign. The description of the DSM algorithm for the case of a linear wave is given in Sect. A1.

The general case of elliptic polarization is sketched in Fig. 3a. In this case, Eqs. (6), (7), (8), added to the three equations which relate $E_{03}, \phi_{3 \mathrm{t}}$ and $\alpha_{3}$ to input quantities, on one hand, and to the equation $\boldsymbol{u v}=0$, on the other hand, do not form a system of linear equations with respect to the unknowns. Consequently, they cannot be easily inversed. We proceed by a "try and test" approach detailed in Sect. A2. The triplet $\left(E_{0}, \phi_{t}, \alpha\right)$ of modulation characteristics measured in a spin plane describes the 2-D properties of the 3D ellipse configuration projected onto the spin plane. Several 3-D ellipse configurations corresponding, after projection, to different quadruplets $\left(m_{a}, m_{b}, \varphi_{a}, \varphi_{b}\right)$ can lead to the same triplet $\left(E_{0}, \phi_{\mathrm{t}}, \alpha\right)$. Starting from the triplet measured on board $\mathrm{C} 4$, we explore the domain of possible values of the angle between meridian planes $P_{4 a}$ and $P_{4 b}$, i.e. we sweep the quantity $\zeta_{4}=2\left(\varphi_{4 a}-\varphi_{4 b}\right)$ in small steps. This provides a series of possible quadruplets $\left(m_{4 a}, m_{4 b}, \varphi_{4 a}, \varphi_{4 b}\right)$, where, in each of them, the positions of $P_{4 a}$ and $P_{4 b}$ meridian planes are known, as well as $m_{4 a}, m_{4 b}$, respectively projections of lengths $a$ and $b$ onto the spin plane. Similarly, we derive a 
series of possible quadruplets $\left(m_{3 a}, m_{3 b}, \varphi_{3 a}, \varphi_{3 b}\right)$ describing the ellipse projected onto $\mathrm{C} 3$ spin plane, from the triplet $\left(E_{0}, \phi_{\mathrm{t}}, \alpha\right)$ measured on board C3. Next, each piece of the $\mathrm{C} 4$ series is paired with each piece of the $\mathrm{C} 3$ series in order to test how well they match. The pairing is based on the approach used for the linear polarization case, where $P_{4 a}$ and $P_{3 a}$ meridian planes intersect along vector $\boldsymbol{u}$, and $P_{4 b}$ and $P_{3 b}$ meridian planes intersect along vector $\boldsymbol{v}$. Knowing zenith angles $\sigma_{a}$ and $\sigma_{b}$ leads to the knowledge of $a$ and $b$ lengths, calculated from $m_{a}^{2}$ and $m_{b}^{2}$ values (in C4 spin plane or in C3 spin plane). A good match must satisfy the constraints fixed by the model of an elliptic polarization:

- $\boldsymbol{u}$ and $\boldsymbol{v}$ are normal to each other,

- a single value of the major semi-axis length a is measured from $\mathrm{C} 4$ or from $\mathrm{C} 3$,

- a single value of the minor semi-axis length $\mathrm{b}$ is measured from $\mathrm{C} 4$ or from $\mathrm{C} 3$.

When there is no satisfying solution, the model is considered not valid. When there are several acceptable solutions, a best one is chosen.

Circular polarization is a specific case of elliptic polarization where the two main axes have the same magnitude and their position in the wave plane can thus be chosen at will. A convenient choice places the major axis $\boldsymbol{u}$ at the intersection of the polarization circle with spin plane, hence aligned with its projection $\boldsymbol{u}_{\mathrm{p}}$ in spin plane (Fig. $3 \mathrm{~b}$ ). As a consequence, the power measured along the major axis of the projected ellipse, i.e. $s_{\max }^{2}$, indicates signal intensity value, $I=2 a^{2}$, whatever inclination of the wave plane with respect to spin plane. Noting that the meridian plane defined by spin axis and antenna position at minimal signal (along the minor axis of the projected ellipse, b $\boldsymbol{v}_{p}$ ) contains vector $\boldsymbol{k}$ (Fig. 3b), we calculate $\boldsymbol{k}$ vector coordinates by intersecting the two relevant meridian planes determined respectively from $\mathrm{C} 3$ and $\mathrm{C} 4$ platforms. This 3-D result, obtained without use of any modulation factor (a quantity which can be affected by the limited sensitivity of receivers), is a clear improvement, compared to the 2-D result of direction finding from a single spacecraft.

\subsection{Performance of the DSM tool}

The inversion algorithm described in the Appendix has been tested by exploring a large range of ellipticity ratio values, $e=b / a$, of $\boldsymbol{k}$ vector orientations, and of $\boldsymbol{u}$ vector orientations, with an excellent return. Overall uncertainties depend on a range of factors, not only uncertainties on the six measured quantities $E_{03}, \alpha_{3}, \phi_{3 \mathrm{t}}, E_{04}, \alpha_{4}$, and $\phi_{4 \mathrm{t}}$, but also on actual characteristics of the ellipse, orientation and ellipticity ratio. A systematic presentation of expected uncertainties in $\boldsymbol{k}$ vector orientations is outside the scope of this paper.

In order to illustrate the quality of direction finding obtained for the chosen event, we take the example shown in
Fig. 1. First, we note that individual samples of measured electric field power densities display a significant variability. The observed fluctuations are not due to galactic or electronic noise, but inherent to the NTC waves under study. They obviously limit the performance which can be obtained with the DSM tool, even after fluctuations are smoothed out by performing a fit between the measured samples and the spin modulation model. In the example shown in Fig. 1, the spherical coordinates of vector $\boldsymbol{u}$ are $\mu_{a}=85^{\circ}$ and $\sigma_{a}=148^{\circ}$ (in the $\left[S_{\mathrm{C} 4}\right]$ coordinate system). For vector $\boldsymbol{v}$, the coordinates are $\mu_{b}=163^{\circ}$ and $\sigma_{b}=97^{\circ}$. The quality of the result is measured by the three quantities listed in the Appendix, $d a, d b$, and $p_{u v}$, equal respectively to $0.026,0.001$ and 0.012 . Observing that $d b$ is small with respect to $d a$ and $p_{u v}$, one can neglect the error on the component $b v$ of the electric field along the small axis of the ellipse. When attributing all quantitative misfits to an uncertainty (or a variability) on vector $\boldsymbol{u}$ orientation, one obtains less than $1^{\circ}$ uncertainty in azimuth, and less than $2.5^{\circ}$ in zenith angle. This result provides an indication of the precision obtained on ray path direction finding for this measurement. Further on (Sect. 4.2), we use statistics to discuss the direction finding performances for the case of the global NTC wave event studied.

We note that the ellipticity ratio obtained $(e=0.87)$ is significantly distant from a circular polarization value $(e=1)$ and cannot be explained by the values obtained for $d a, d b$ and $p_{u v}$ parameters. We examine below how the classical assumption of a purely circular polarization (usually imposed when a single spacecraft is available) can impair the correct estimation of wave normal orientation.

\section{Application of DSM tool}

\subsection{Output of the DSM tool: discussion of a selected case}

The DSM tool delivers two outputs, the modelled signals (electric field intensity versus antenna attitude) under respectively circular and elliptic polarization assumptions. Let us see, as an illustration, what is obtained by the DSM tool for the one minute time interval presented in Fig. $1 \mathrm{~b}$ and c. Figure 4 shows again the signal best fit to actual measurement, plotted in green dashed lines (for C3) and blue dashed lines (for $\mathrm{C} 4$ ). It takes account of the correction factor needed from onboard spectra accumulation, explaining the divergence with actual measured intensities (gray crosses) near extrema of curves. Outputs from DSM tool are plotted in red solid lines under the assumption of elliptical polarization, and in red dashed lines under the assumption of circular polarization. Under a circular polarization assumption, the maximal values of electric field power, $s_{\max }^{2}$, measured independently from $\mathrm{C} 3$ and from $\mathrm{C} 4$, should be equal to each other. Actual $s_{\max }^{2}$ values (in unit $10^{-12} V_{\mathrm{rms}}^{2} \mathrm{~Hz}^{-1}$ ) are 39 and 31.5, as measured respectively on $\mathrm{C} 3$ and $\mathrm{C} 4$. Those values could be considered to be equal, with a common 
2008/05/18 12:20:00 UT (60s) / 15.0 - $24.7 \mathrm{kHz}$
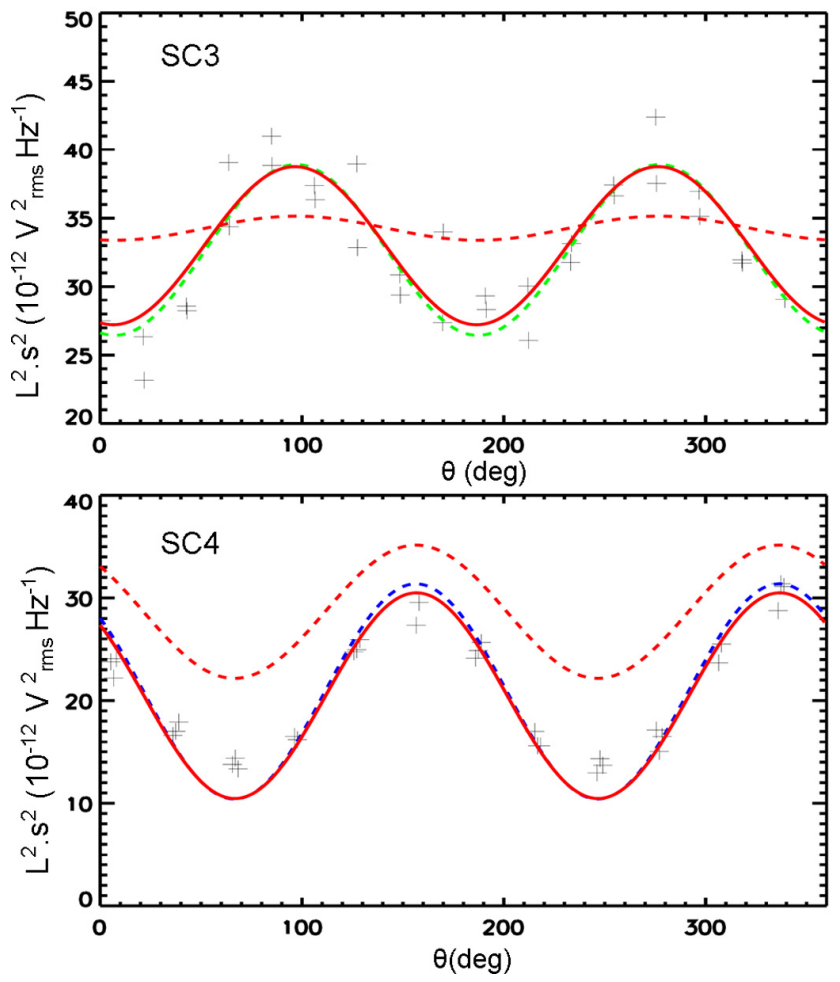

Fig. 4. Electric signal versus antenna attitudes, with $\mathrm{C} 3$ data at top and C4 data at bottom (same data set as in Fig. 1). As in Fig. 1, grey crosses are measured samples, the dashed green curve (top) and dashed blue curve (bottom) represent best fits to instantaneous signal variations. Red curves correspond to the modelled electric fields (instantaneous power variations) solution of the DSM tool under respectively elliptical polarization assumption (solid lines) and circular polarization assumption (dashed lines).

$s_{\max }^{2}$ value of $35.25 \mathrm{~V}_{\mathrm{rms}}^{2} \mathrm{~Hz}^{-1}$, accounting for global measurement uncertainties of $20 \%$ on the electric field magnitude (Trotignon et al., 2010). However, a survey of the wave power at phase zero over a large time and frequency domain (Sect. 4.2) shows that C3 and C4 WHISPER instruments are almost perfectly cross calibrated during this event, when they share the same environment, indicating that the signal levels measured are reliable. In conclusion, Fig. 4 is a convincing illustration that, when considering $\mathrm{C} 3$ and $\mathrm{C} 4$ modulation parameters as describing the same wave, (i) the assumption of elliptical polarization fits very well the measured double spin modulation (red solid lines compared to green and blue dashed lines); and (ii) the assumption of circular polarization fits poorly the measured double spin modulation (red dashed lines compared to green and blue dashed lines).

To conclude the analysis of this one minute time interval, the spin modulations measured from the tilted Cluster pair over the chosen frequency band $(15-25 \mathrm{kHz})$ are remarkably consistent with the model of a plane wave where the electric field is elliptically polarized. Let us call $\boldsymbol{k}_{E}$ the vector $\boldsymbol{k}$ (normal to wave plane) derived with this model. The measured magnitude at major axis is $9.0 \times 10^{-6} V_{\mathrm{rms}} \mathrm{Hz}^{-1 / 2}$, the ellipticity ratio $e=0.87$, and the orientation of vector $\boldsymbol{k}_{E}$ is (in GSE coordinates and with a $180^{\circ}$ ambiguity) $120^{\circ}$ in zenith angle $v$ (angle from $Z_{\mathrm{GSE}}$ axis to $\boldsymbol{k}_{E}$ ) and $127^{\circ}$ in azimuth $\phi$ (angle from $X_{\mathrm{GSE}}$ axis to $\boldsymbol{k}$ projected onto $X_{\mathrm{GSE}} Y_{\mathrm{GSE}}$ plane). The modelled spin variations under a circular polarization assumption poorly reflect power variations versus attitude. However, the components of vector $\boldsymbol{k}$, which we shall call $\boldsymbol{k}_{\mathrm{C}}, 128^{\circ}$ in zenith angle and $130^{\circ}$ in azimuth, and the signal magnitude, equal to $12 \times 10^{-6} V_{\mathrm{rms}} \mathrm{Hz}^{-1 / 2}$, are quantities crudely similar to results obtained with the former model.

\subsection{Direction finding: critical evaluation of the single spacecraft approach}

\subsubsection{Case event of 18 May 2008, 12:20-12:21 UTC time interval}

We compare in more detail the results of direction finding from a single spacecraft, DF1 (under assumption of circular polarization), with results of direction finding from the tilted pair, DF2 presented above. We assume that the tilted pair provides a correct estimation of the polarization ellipse and of the unit wave vector $\boldsymbol{k}$, called $\boldsymbol{k}_{E}$ when derived from DF2. We simply compare the orientation of $\boldsymbol{k}_{\mathrm{C} 1}$, unit wave vector derived from DF1 (using data of C4 or C3, successively), against the orientation of $\boldsymbol{k}_{E}$. We start the analysis by comparing wave vector directions in the spin plane (which is the information provided by the DF1 approach).

The sketch of Fig. 3a shows the general case, here for directivity analysis from $\mathrm{C} 4$ viewpoint, the configuration of the electric field ellipse and of the wave vector $\boldsymbol{k}$, both projected onto the spin plane. In this sketch, the antenna is shown when aligned with the minor axis of the projected ellipse (its position when it measures minimal signal intensity). This orientation generally differs from that of $\boldsymbol{k}_{\mathrm{p}}$, the projected wave vector $\boldsymbol{k}$. In other words, the ray path orientation in spin plane derived in the DF1 approach is identified by the azimuth $\phi_{\mathrm{m}}$ (with respect to the reference axis) at minimum signal intensity, whereas the projection of the vector $\boldsymbol{k}_{E}$ ( $\boldsymbol{k}$ in the sketch) onto spin plane corresponds to an azimuth value $\phi_{\mathrm{e}}$. The difference $\phi_{\mathrm{m}}-\phi_{\mathrm{e}}$ measures the error in azimuth orientation resulting from using the assumption of circular polarization. In the case of $\mathrm{C} 4$, where the modulation factor $\alpha$ is 0.50 for the selected case event, the difference $\phi_{\mathrm{m}}-\phi_{\mathrm{e}}$ is small, equal to $2.3^{\circ}$ in absolute value. In the case of satellite $\mathrm{C} 3$, where the modulation factor $\alpha$ is 0.19 , the difference $\phi_{\mathrm{m}}-\phi_{\mathrm{e}}$ is much larger, equal to $24^{\circ}$. The general study presented below indicates that errors in azimuth orientations in spin planes are indeed increasing with decreasing modulation factor $\alpha$.

One can take further the exploitation of measured parameters within the frame of the DF1 approach, and derive $\boldsymbol{k}$ vector orientations in 3-D by using the relationship between 
modulation factor $\alpha$ and $\boldsymbol{k}$ elevation angle $\eta$ with respect to spin plane by

$$
\sin ^{2} \eta=\frac{1-\alpha}{1+\alpha} .
$$

Equation (9) is developed in Grimald et al. (2011), using a slightly different notation. It is equivalent to the relation $\cos ^{2} \eta=m$ published in Gurnett (1975). In any event, Eq. (9) (or its equivalent) indicates that quantities $\eta$ and $\alpha$ are strongly linked to each other and that knowledge of the modulation leads to two possible solutions for $\eta$, corresponding to $\boldsymbol{k}$ vectors symmetric with respect to the spin plane. This ambiguity, added to the $180^{\circ}$ ambiguity in ray path direction, complicates the search for source location. In our selected case event, we simply choose the solution providing a final orientation of $\boldsymbol{k}$ as close as possible to the true orientation. Let us call $\boldsymbol{k}_{\mathrm{C} 1}$ this estimation of $\boldsymbol{k}$. We characterize the orientation of $\boldsymbol{k}_{\mathrm{C} 1}$ in GSE coordinate system by the zenith angle $v$ and the azimuth $\phi$ (which differs from the azimuth at minimum electric field intensity derived in the spin plane). When derived from $\mathrm{C} 4, \boldsymbol{k}_{\mathrm{C} 1}$ is oriented at zenith and azimuth angles respectively of 124 and $130^{\circ}$ (compared to 120 and $127^{\circ}$ for $\boldsymbol{k}_{E}$ ), hence within a cone angle of $5^{\circ}$ from $\boldsymbol{k}_{E}$. When derived from $\mathrm{C} 3, \boldsymbol{k}_{\mathrm{C} 1}$ is oriented at zenith and azimuth angles respectively of 119 and $146^{\circ}$, hence within a cone angle of $18^{\circ}$ from $\boldsymbol{k}_{E}$. In order to complete the comparison, we can summarize that $\boldsymbol{k}_{\mathrm{C} 1}$ derived from $\mathrm{C} 4$ is placed within a cone angle of $5^{\circ}$ from $\boldsymbol{k}_{\mathrm{C}}$ (the unit wave vector derived from the DF2 approach under approximation of a circular polarization) and that $\boldsymbol{k}_{\mathrm{C} 1}$ derived from $\mathrm{C} 3$ is placed within a cone angle of $17^{\circ}$ from $\boldsymbol{k}_{\mathrm{C}}$.

\subsubsection{Expected performance with a single spacecraft, general case}

Let us consider a given elliptical configuration of the electric field, linked to unit vector $\boldsymbol{k}_{E}$, creating spin modulation characteristics $E_{0}, \alpha$, and $\phi_{\mathrm{m}}$ measured from a single spacecraft. Those characteristics, interpreted within the frame of the "spin-null method", indicate the orientation $\boldsymbol{k}_{\mathrm{C} 1}$ of wave normal. We first consider errors in azimuth directivity angles between $\boldsymbol{k}_{\mathrm{C} 1}$ and $\boldsymbol{k}_{E}$ (the latter assumed to be the true unit wave vector). We define the azimuth error angle $\varepsilon=\left(\phi_{\mathrm{m}}-\phi_{\mathrm{e}}\right)$, where $\phi_{\mathrm{m}}$ and $\phi_{\mathrm{e}}$ are the azimuths of respective $\boldsymbol{k}_{\mathrm{C} 1}$ and $\boldsymbol{k}_{E}$ projected onto spin plane.

In order to evaluate the quantity $\varepsilon$ in the general case, we characterize the electric field ellipse by its shape and by its configuration in the coordinate system attached to the measuring spacecraft. The shape is defined using the ellipticity ratio $e=b / a$, and the configuration is characterized by two quantities, the angle $\eta$ of elevation of normal vector above spin plane, on one hand, and on the other hand the angle $\lambda$ of major axis, in wave plane, with the intersection between wave plane and spin plane (sketch at top left of Fig. 5). For a given magnitude and orientation of the major and minor axis of the ellipse, $a \boldsymbol{u}$ and $b \boldsymbol{v}$, we calculate the characteristics of the modulated signal obtained in the spin plane by adding the contributions of the two oscillating components aligned with $\boldsymbol{u}$ and $\boldsymbol{v}$ (see the Appendix). We then derive the desired quantities. The right side panels in Fig. 5 display the results of our analysis under a large ellipticity ratio, $e=0.85$, a value close to that of our case event, and under a smaller value, $e=0.7$. The azimuth error angle $\varepsilon$ versus configuration angle $\lambda$ is plotted as solid lines, colour coded according to elevation angle values $\eta$ (colour code indicated in right side panels). The error angle $\varepsilon$ varies with ellipse configuration, as expected, and can reach unacceptably large values, up to $90^{\circ}$. This result had been foreseen by Jones $(1982,1983)$.

The value of modulation factor $\alpha$, which is displayed in dashed lines in the same panels, could be used to select cases with an acceptable error angle $\varepsilon$. Selecting, for instance, large modulations, like $\alpha>0.8$, would secure an error below a threshold possibly acceptable, about $5^{\circ}$ in absolute value (estimated when following dark blue dashed lines in both panels at right side of Fig. 5, and considering corresponding error values). Pushing ellipticity ratio e at lower values (results not shown) indicates that the same selection criteria $\alpha>0.8$ would not secure a good direction finding (the error angle $\varepsilon$ can reach about $20^{\circ}$ for $e=0.5$ ). In practice, the modulation factor of NTC radiation measured on board Cluster being most often smaller than 0.8 (see also results on escaping continuum observed on IMP-8 by Gurnett, 1975), the polarization dependency on directivity estimation has to be taken seriously.

The bottom left panel in Fig. 5 displays the ray path elevation angle (ordinate) estimated from modulation measured on a single satellite (abscissa) and interpreted within the frame of circular polarization (Eq. 4). This curve can be used to get an idea of the error when deriving $\boldsymbol{k}$ direction in 3-D under circular polarization. For $e=0.85$, for instance, a given elevation $\eta$ and varying angle $\lambda$, the actual modulation value $\alpha$ (shown in the top right panel) explores a significant interval, corresponding to a significant portion of the curve plotted in bottom left panel. This portion is highlighted by a rectangle in the colour referring to the given $\eta$ (for instance, light blue colour in the case of an elevation $\eta=35^{\circ}$, where the $\alpha$ interval is [35-60\%] and the corresponding $\eta$ interval is $\left.\left[31-42^{\circ}\right]\right)$. As an example, let us suppose that the true elevation is $35^{\circ}$ and $e=0.85$. The measured $\alpha$ value is anywhere between 35 and $60 \%$; leading to an estimated $\eta$ between 31 and $42^{\circ}$, leading to the error interval -4 to $+7^{\circ}$. Distances from the true elevation increase with the chosen elevation value, from $\sim \pm 5^{\circ}$ at $\eta=20^{\circ}$, to $\sim\left[-30^{\circ},+20^{\circ}\right]$ at $\eta=65^{\circ}$. Nevertheless, the chance to obtain the correct value are non-zero, since all rectangles contain the true $\eta$ value. For $e=0.7$, however, and $\eta=65^{\circ}$, the domain of estimated ray path elevation (rectangle in dashed lines, orange colour) does not contain the true $\eta$ value, the estimation being severely misleading. 

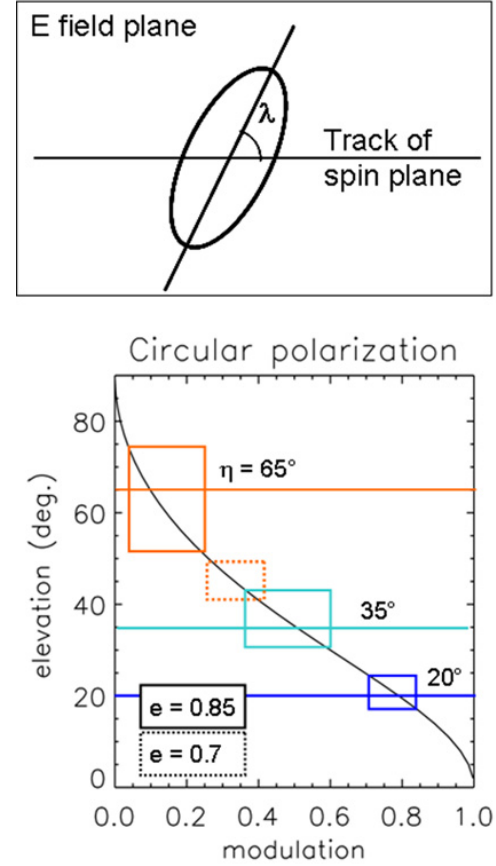
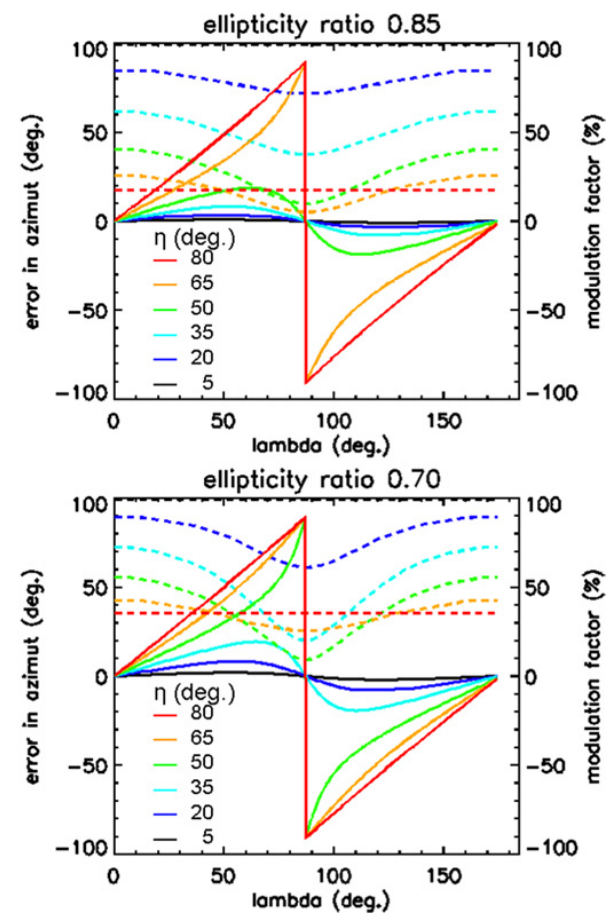

Fig. 5. Top left: sketch of ellipse in electric field plane, displaying the angle $\lambda$ between the intersection of the ellipse with the spin plane and the major axis. Right: Azimuth error in direction finding as a function of major axis orientation angle $\lambda$ (solid lines). Two values of ellipticity ratio are examined (top and bottom panels, respectively) and six values of elevation $\eta$ (indicated by colour codes). Dashed lines show variations with angle $\lambda$ of measured modulation factor $\alpha$. Bottom left: the black solid line displays elevation angle $\eta$ of $\boldsymbol{k}$ vector versus modulation factor $\alpha$ under circular polarization $(e=1)$. Rectangles delimit domains of $\eta$ values derived from measured modulation and assuming $e=1$ when $e<1$; in solid lines at $e=0.85$ for three values of $\eta\left(65,35\right.$ and $\left.20^{\circ}\right)$ and in dashed lines at $e=0.7$ for $\eta=65^{\circ}$.

In conclusion, this study indicates that direction finding with a single spacecraft should be taken with caution. It is valid only in the case of circular polarization or within the frame of a quasi-circular polarization when a high modulation factor is measured. Less restrictive applications could lead to very large deviations, and explain puzzling wave source positions obtained that way. One must be particularly cautious when using directivity angles to derive a source position by triangulation, as illustrated in Sect. 5 .

\section{NTC observations and directivity analysis}

\subsection{Observations}

During the tilt campaign (May 2008), several radio wave events were observed by WHISPER instruments. We choose to analyse and present here an event observed on 18 May 2008, from 11:40 to 13:00 UTC, with a spectral signature similar to the "wide-banded" type of NTC emissions (Masson et al., 2009). The global geomagnetic context is shown in Fig. 6a, which displays geomagnetic indices am (red line) and ap (black line) during a two week time interval. The am series allow, with an extended coverage of the associated magnetometers network, the detection of small perturbations (of interest during quiet periods) better than the ap series does (Menvielle and Berthelier, 1991). The event (start time shown by a pink vertical bar) occurs after a very quiet day (ap $\leq 3 \mathrm{nT}$, i.e. $\mathrm{Kp}<1$, on 17 May), preceded by a 10 days interval (7 May to 16 May) of quiet or moderate activity (ap $\leq 7 \mathrm{nT}$, i.e. $\mathrm{Kp} \leq 2$ ). The interplanetary and geophysical context nearer the event, characterized by a surge of auroral activity between 11:00 and 12:00 UTC on 18 May, is presented in Fig. 10 and discussed in Sect. 5.3. The Cluster constellation is travelling above the polar cap, at a geocentric distance of about $8 R_{\mathrm{E}}$. The sketch in Fig. $6 \mathrm{~b}$ indicates the positions of the satellites at 12:20 UTC, with C1 in black, C2 in red, and the tilted pair, $\mathrm{C} 3$ and $\mathrm{C} 4$, in green. The baseline formed by the three satellites is an elongated triangle, about parallel to the ecliptic plane, shown in Fig. 8.

The chosen event displays a spectral signature outlined by an orange oval shape in the frequency time spectrogram of Fig. 6c. It bears the fundamental characteristic of wide-banded NTC emissions, i.e. quasi-harmonic frequency bands, separated one from the next by the same frequency step, here $d f \sim 2.5 \mathrm{kHz}$. The df parameter is stable during the time interval 11:50 to $12: 40$ UTC, outlined by the two vertical purple arrows. In contrast, local plasma parameters are varying along the orbit element followed during 


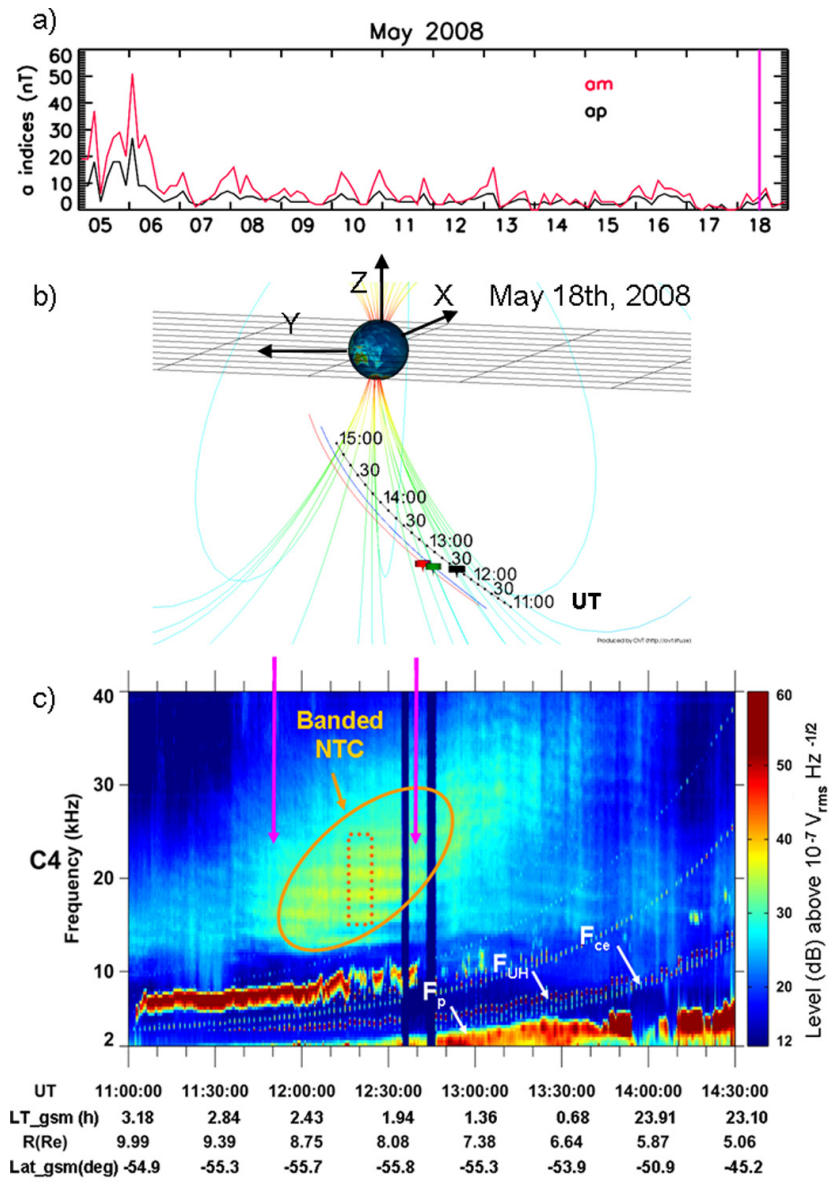

d)

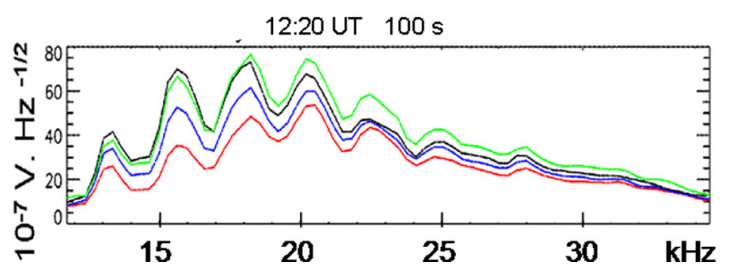

Fig. 6. NTC emissions observed on 18 May 2008 from Cluster constellation. (a) $3 \mathrm{~h}$ planetary indices in a two week interval prior and during the event (starting at purple vertical line). (b) Cluster constellation on its inbound pass, in GSM coordinate system at 12:20 UTC. Cylinder-like symbols indicate satellite positions, respectively in black for $\mathrm{C} 1$, red for $\mathrm{C} 2$, and green for the tilted pair C3-C4. (c) Spectrogram from WHISPER instrument on board $\mathrm{C} 4$. Vertical arrows point to the time interval of prominent NTC emissions. Sounder resonances and natural wave signatures measure local characteristic frequencies, gyro-frequency $F_{\text {ce }}$, plasma frequency $F_{\mathrm{p}}$, and upper hybrid frequency, $F_{\mathrm{UH}}$, pointed by white arrows. Intense emissions at $\sim 6-8 \mathrm{kHz}$, observed from about 11:00 to $12: 40$ UTC, are spurious oscillations under investigation. (d) Electric field frequency spectra measured on the four satellites at 12:20 UTC, averaged over a $100 \mathrm{~s}$ time interval. Intensity variations are shown in black for $\mathrm{C} 1$, red for $\mathrm{C} 2$, green for $\mathrm{C} 3$ and blue for C4. the quoted time interval. They can be measured by the frequency positions of resonances triggered by the sounder within its frequency band, i.e. $4-80 \mathrm{kHz}$. Having noted that intense emissions at $\sim 6-8 \mathrm{kHz}$, observed from about 11:00 to 12:40 UTC, are spurious oscillations, the identified characteristic frequencies are the electron gyro-frequency $F_{\text {ce }}$, the upper hybrid frequency $F_{\mathrm{UH}}$, and the derived plasma frequency $F_{\mathrm{p}}$, each pointed to by a white arrow in Fig. 6c. The local magnetic field magnitude increases significantly during the outlined time interval. The associated electron gyro-frequency $F_{\text {ce }}$ increases from $2.5 \mathrm{kHz}$ at 11:50 UTC to $3.7 \mathrm{kHz}$ at 12:40 UTC. The gyro-frequency is identified by sounder resonances at twice $F_{\text {ce }}$, observed above WHISPER sounder's low frequency limit. The electron density is measured by the frequency position of $F_{\mathrm{p}}$, which increases during the chosen interval from below $2 \mathrm{kHz}$ to about $2.7 \mathrm{kHz}$, all frequencies significantly below the frequency range, $\sim 15$ to $25 \mathrm{kHz}$, covered by the main NTC bands. All four satellites are illuminated simultaneously by the radio source of the observed NTC emissions. This is illustrated in Fig. 6d by the remarkable similitude of spectral signatures measured on board the four Cluster satellites, here integrated over the $100 \mathrm{~s}$ time interval starting at 12:20 UTC. Figure $6 \mathrm{~d}$ points out that the observed average intensity is significantly higher on $\mathrm{C} 3$ than on $\mathrm{C} 4$. This difference in intensities is also clearly seen in power variations shown in Fig. 1. The reason for such a discrepancy is that the wave plane makes a smaller angle with C3 spin plane than with C4 spin plane. It confirms that the wave is planar. Wave intensities are also gradually increasing when observed respectively from $\mathrm{C} 2, \mathrm{C} 4$ and $\mathrm{C} 1$. This feature will be discussed later (end of Sect. 5.3.2).

Frequency-time spectrograms similar to the example displayed in Fig. 6c are actually rather common in Cluster data, when the formation travels within low density regions of magnetosphere. They had been observed already during earlier explorations (Gurnett and Shaw, 1973). The value of local plasma frequency during the time interval of our observation, well below the frequency range of main bands, offers the context needed to undertake a study within the frame of the free propagation assumption. We assume that the source of the radiation is placed at a steep gradient in plasma frequencies (covering the $15-25 \mathrm{kHz}$ range), which favours the emission of NTC radio waves. We assume also a narrow connection between the band frequency separation and the gyrofrequency local to the source, a feature initially emphasized by Gough (1982). This important quantitative marker actually forms the basis of the source search described in Sect. 5 .

\subsection{Directivity analysis}

The 3-D directivity analysis performed by the DSM tool presented in Sect. 3.1 indicates that, in the example selected, electric field measurements from the tilted pair of $\mathrm{C} 3$ and $\mathrm{C} 4$ spacecraft are representative of a plane wave, elliptically polarized. They are, in any case, displaying spin modulations 

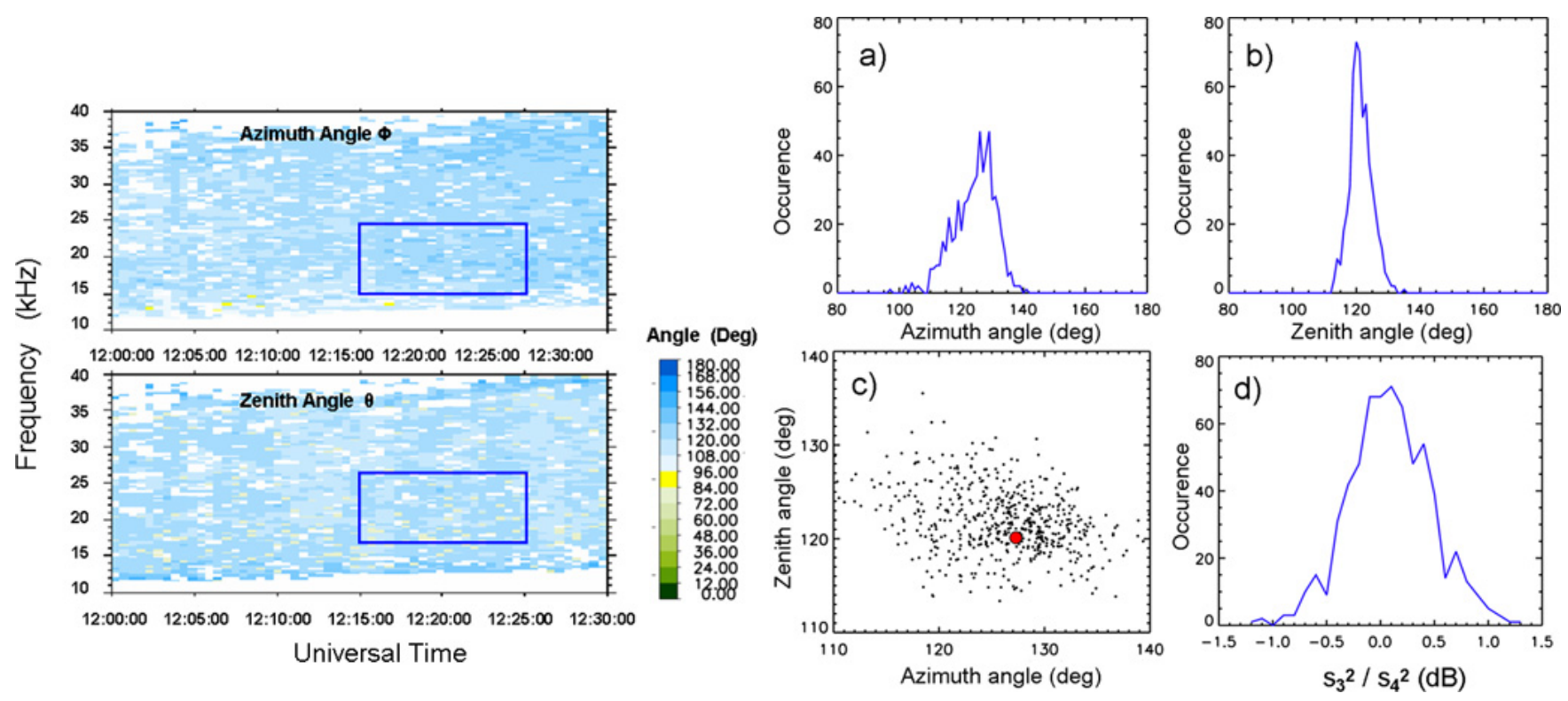

Fig. 7. DSM tool applied to a frequency-time domain during 18 May 2008 event. Elementary runs treat the signal at a fixed frequency bin (320 Hz width) measured on C3 and C4 during an interval of $60 \mathrm{~s}$. Left: frequency-time spectrograms of derived ray path vector orientation angles (azimuth and zenith) in GSE coordinate system. Right: distribution of quantities derived from the runs inside the pointed frequencytime domain (blue rectangle); (a) azimuth angle occurrence, (b) zenith angle occurrence, (c) zenith versus azimuth, and (d) ratio of electric field powers measured on $\mathrm{C} 3$ and $\mathrm{C} 4$ along the common antenna orientation.

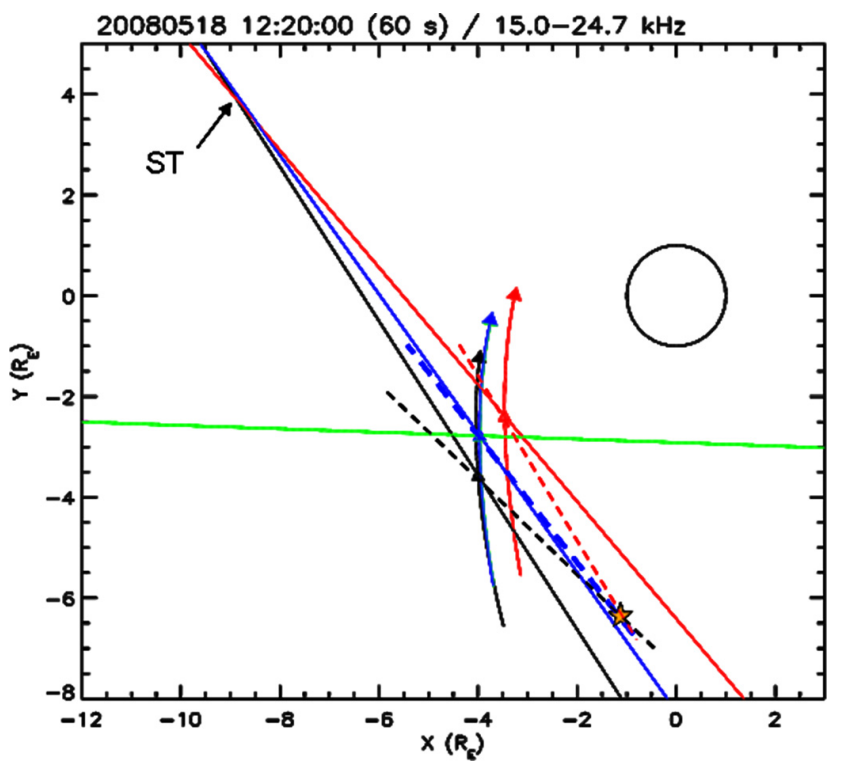

Fig. 8. Source localization by triangulation for the event of 18 May 2008. Orbit elements (curved arrows) and ray paths (solid lines) of a given colour intersect at satellite positions (black for $\mathrm{C} 1$, red for $\mathrm{C} 2$, green for $\mathrm{C} 3$ - see text - and blue for $\mathrm{C} 4$ ), ray paths merging at source position from triangulation, ST, pointed by an arrow. This plot also displays the position of source (orange star) obtained from the 3-D-isoFce approach (see text and Fig. 9), dashed lines showing associated source to satellite ray paths projected unto the $X Y$ GSE plane. expected in such a circumstance. The next question we address is whether the ray path orientation obtained by the analysis is constant over the frequency and time domain covered by the NTC emissions observed during the event.

First of all, we note that the result of DSM analysis presents a significant variability in response to the precise input taken into account, a data set which always consists of signal intensities versus phase angles (as shown in Fig. 4). Two parameters of the input data set are adjustable: the number of measured intensity samples (duration of considered time interval) and the frequency band over which measured intensities are averaged (i.e. the number and position of relevant frequency bins). The output of the DSM tool is actually responsive to small variations in the input introduced by varying any one of the two adjustable parameters. The individual orientations obtained for the bins where signal to noise ratio is acceptable are, however, gathered around the orientation obtained when directly averaging intensities over the frequency band. In order to further examine the response of the DSM tool during the chosen NTC event, we have constructed frequency-time spectrograms shown in Fig. 7 (left panels). These spectrograms are built from elementary runs of the DSM tool (electric fields measured on $\mathrm{C} 3$ and $\mathrm{C} 4$ spacecraft at a fixed frequency bin, during a given time interval of $60 \mathrm{~s}$ ). Each run delivers, when the analysis is successful, the ray path orientation in the form of two angles, azimuth and zenith. The calculation is done, for the same time inter$\mathrm{val}$, on each of the frequency bins covering the $10-40 \mathrm{kHz}$ band, and repeated at later time intervals. Time frequency 
spectrograms of azimuth angles (top panel) and zenith angles (bottom panel) display bluish colours (coding angle values) roughly similar over the overall frequency and time domain explored. Patches of white correspond to frequencytime positions where the analysis is unsuccessful. This happens under various circumstances: (i) when NTC emissions are not observed, in particular at low frequency, as shown by the clear boundary observed in the spectrograms at $\sim 12-$ $13 \mathrm{kHz}$, precisely at the low frequency cut-off of emissions (see Fig. 6); (ii) when modulation parameters cannot be calculated, i.e. when input signal to noise ratio are below a given threshold; and (iii) when the modulation parameters cannot be modelled by a planar elliptic wave, as described in Sect. 2.3 (no satisfying candidate solutions).

Having found no clear evolution of directivity results with frequency and time, at least within the frame of instrument performance, we wish to estimate a single ray path orientation representative of the global behaviour of observed waves. To this aim, we present statistics limited to a frequency-time domain chosen in the centre of the event. This domain, underlined by rectangles in Figs. $6 \mathrm{c}$ and 7 (orange dashed lines and blue lines, respectively) is the 15$25 \mathrm{kHz}$ frequency range, and the 12:15-12:25 UTC time interval. This is covered by 620 individual runs of the DSM tool: 20 iterations in time ( $60 \mathrm{~s}$ duration, $30 \mathrm{~s}$ increment) and 31 iterations in frequency ( 31 bins). Corresponding results are shown in the right-hand panels of Fig. 7: occurrences of orientation angles are plotted in panels (a) and (b), and the corresponding scatter plot in panel (c).

The last view derived from our statistical analysis (Fig. 7d) shows the occurrence of signal intensities ratios when measured at the same antenna attitude (at phase zero) respectively from $\mathrm{C} 3$ and $\mathrm{C} 4$. It allows us to check whether the two WHISPER instruments on board C3 and C4 satellites are equivalent. First simulations have shown that a high absolute precision for physical values is needed to derive ellipse characteristics, better than global uncertainty intervals on gains derived from ground integration campaigns (i.e. $4 \mathrm{~dB}$ ) would warrant. What is the acceptable tolerance? Is it satisfied during a limited time interval (when the environment is common)? It is not possible to directly compare electric field intensities measured along the same direction from the two close-by satellites, C3 and C4, since antennas are never aligned at the same time along the common direction they sweep (the intersection of spin planes), but the DSM tool analysis offers an indirect comparison. It allows to estimate electric field intensities at phase zero independently on $\mathrm{C} 3$ and $\mathrm{C} 4$ for each sample of the time-frequency domain examined (the 620 individual runs described above). The curve of Fig. 7d displays the occurrence of ratio $s_{3}^{2} / s_{4}^{2}$ determined at each run of DSM tool within the time-frequency domain chosen (most of them successful), where $s_{3}^{2}$ and $s_{4}^{2}$ are modelled intensities (i.e. the sine function best fitted with actual signal variations) at zero phase angles. The occurrence curve culminates at a ratio value $s_{4}^{2} / s_{3}^{2}$ corresponding to $0.1 \mathrm{~dB}$, and ratios at half width correspond to $0.5 \mathrm{~dB}$. We estimate that the dispersion in occurrence values can be explained by the fluctuations observed in raw measurements. In order to further check the consequence of the limited precision regarding instrument gains on the 3-D directivity analysis, we have quantitatively investigated what the angle deviation is between orientations obtained with the current $\mathrm{C} 3$ calibration function, and with a calibration function at a higher gain, which would place the ratio value $s_{4}^{2} / s_{3}^{2}$ at $0 \mathrm{~dB}$ for the highest occurrence shown in Fig. 7d. We obtain a negligible angular deviation $\left(0.5^{\circ}\right)$ with a $0.1 \mathrm{~dB}$ gain increase and a small one $\left(\sim 2^{\circ}\right)$ with a $0.5 \mathrm{~dB}$ gain increase. We conclude that no calibration tuning is necessary.

The distribution of orientation angles shown in the right side panels of Fig. 7, panels (a) and (b), display clear peaks at both azimuth and zenith orientation angles, a double peak around azimuth $\phi=127^{\circ}$ and a single peak at zenith $v=$ $120^{\circ}$. This particular orientation, indicated by a red dot in Fig. $7 \mathrm{c}$, is the same one as that found at the centre of the time interval chosen for statistical analysis, which is the selected case example shown in Fig. 4 and discussed in Sect. 3.2. Angles at half width of occurrence distribution functions are (i) $121^{\circ}$ and $132^{\circ}$ in the case of azimuth angles, i.e. at angles of about $5^{\circ}$ from central value, and (ii) $118^{\circ}$ and $123^{\circ}$ in the case of zenith angles, i.e. at angles of about $2.5^{\circ}$ from central value.

\section{Source localization}

\subsection{Strategy}

Two possible approaches can be used in searching for wave source position. The first one is triangulation. Ray paths, traced backward from either two (or more) distant observatories, or from the same observatory travelling along its orbit, intersect at the source. This technique has been used (under assumption of free propagation in its simplest version, where the ray path is a straight line) with various spacecraft fleets (Gough, 1982; Etcheto et al., 1982; Parrot et al., 2004; Grimald et al., 2007, 2011). Figure 8 illustrates the triangulation technique applied to the one minute time interval measurements, shown in Figs. 1 and 4, completed by $\mathrm{C} 1$ and $\mathrm{C} 2$ measurements. The figure displays ray paths observed from Cluster spacecraft (solid lines in black for $\mathrm{C} 1$, red for $\mathrm{C} 2$ and blue for C4) projected onto XY GSE plane. C1, C2 and C4 positions are projected onto the same plane. Ray path orientations are derived within the frame of circular polarization and assuming that spin planes are parallel to $X Y$ GSE plane. Orbit segments near the time of observation are plotted as curved arrows, and satellites (triangular symbols) are placed at the intersection of orbit segments with ray paths of a given colour. Colours used (black, red, green and blue) refer respectively to $\mathrm{C} 1, \mathrm{C} 2, \mathrm{C} 3$ and $\mathrm{C} 4$. C3 orbit element cannot be distinguished from that of $\mathrm{C} 4$ at this scale, and 
the associated calculated ray path (solid green line) should be discarded as $\mathrm{C} 3$ spin plane makes a significant angle with $X Y$ GSE plane. We note that $\mathrm{C} 1, \mathrm{C} 4$ and $\mathrm{C} 2$ form an elongated triangle almost parallel to $X Y$ GSE plane (with respective $Z_{\mathrm{GSE}}$ coordinates $Z_{1}=-6.76 R_{\mathrm{E}} ; Z_{4}=-6.75 R_{\mathrm{E}}$ and $Z_{2}=-6.70 R_{\mathrm{E}}$ ). The ray paths point to a common position (ST), which is thus a candidate source position, here placed in the dusk sector, at large radial distance from Earth's centre and from the constellation $\left(\sim 9 R_{\mathrm{E}}\right.$ and $8 R_{\mathrm{E}}$, respectively). The pointed position actually defines a column segment, perpendicular to $X Y$ GSE plane. Information provided by measured modulation factors, $0.48,0.50$ and 0.50 respectively for $\mathrm{C} 1, \mathrm{C} 2$ and $\mathrm{C} 4$, yields in addition (case of circular polarization, Fig. 5, bottom left panel) ray path elevation angles values around $35^{\circ}$. The radio source would then be placed roughly at $\pm 5.5 R_{\mathrm{E}}$ from the constellation plane. However, the position of the source region obtained in that way is far from the boundaries considered to be where NTC generation occurs (plasmapause, possibly magnetopause). Moreover, the study in Sect. 3.3 has shown that small deviations from circular polarization lead to systematic errors on ray path orientation, requiring being cautious. This problem has a more severe impact under situations such as the current one, where ray paths orientations in 2-D are not far from each other.

Gough (1982) proposed an alternative approach in order to obtain NTC wave source positions. Spectral properties of the waves, assumed to be unchanged from source to observation (as expected within the frame of free propagation), act as a marker of gyro-frequency value local to the source. A given gyro-frequency (associated to a given magnetic field magnitude) fixes the corresponding region of magnetosphere, which we shall refer to in this paper as an isoFce surface. When the terrestrial magnetic field is modelled by that of a dipole, isoFce surfaces are flattened spheres, as indicated in Fig. 9, which displays sections of isoFce surfaces in a meridian plane (dashed green lines). The source position is then determined by the intersection of the ray path, traced back from a single spacecraft, with the relevant surface. The main difficulty encountered in several published applications of this strategy (Gough, 1982; Décréau et al., 2004) is the fact that the ray path orientation is known only in a plane. This difficulty disappears when the ray path orientation is known in 3-D, as shown by successful mapping of AKR radiation sources using DE-1 observations (Huff et al., 1988), or, more recently, of SKR radiation sources using Cassini observations (Cecconi et al., 2009). This second strategy is hence the favoured one in the event analysed. Its application is presented in detail below. As this approach combines ray path orientation (via directivity) and selection of a given magnetic intensity surface (via spectral signatures), we shall refer to it as the 3-D-isoFce approach.

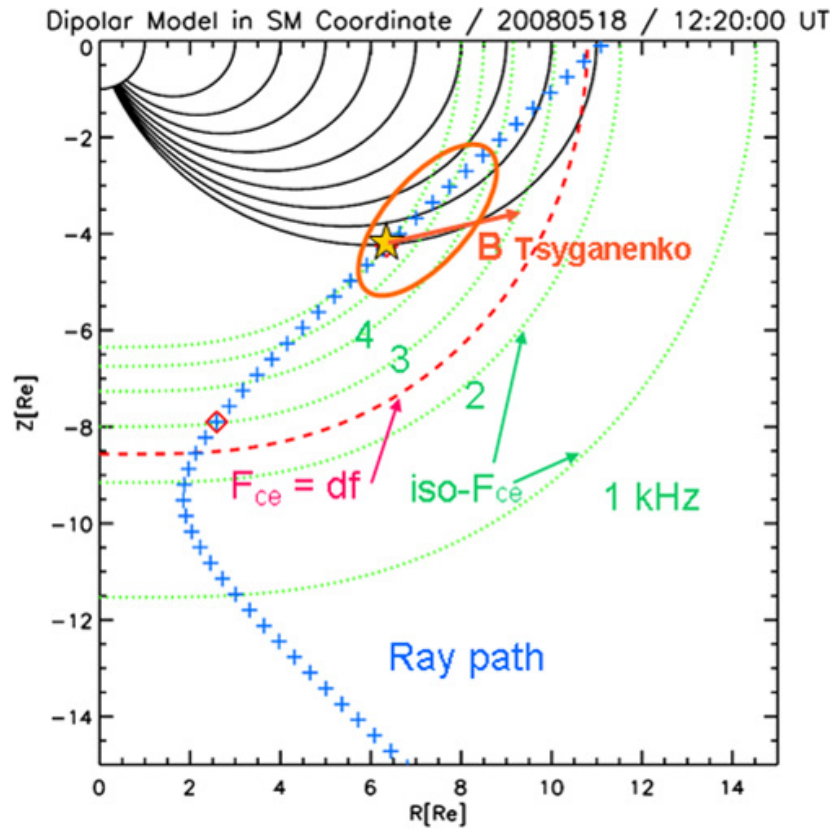

Fig. 9. NTC source position on 18 May 2008, obtained by the 3-DisoFce approach and displayed in a meridian view of SM coordinate system $(R$ : distance from dipole axis; $Z$ : distance from magnetic equatorial plane). Cluster tilted pair is represented by a red diamond and the ray path by blue crosses. Curves at constant gyro-frequency (according to magnetic dipole model) are plotted in green for round $F_{\text {ce }}$ values and in dashed red for the gyro-frequency value at source (as estimated from NTC signature). The source position in a realistic magnetic field model is shown by a star, inside a global region sketched by an oval (see text), and the orange arrow points to the modelled magnetic field vector $\boldsymbol{B}$ at source.

\subsection{Localization of sources in 3-D}

The first element of the 3-D-isoFce approach is to follow the ray path backward from the $\mathrm{C} 3-\mathrm{C} 4$ observatory. As the ray path direction is known, but not its sign, two opposite directions have to be explored, away from and toward equatorial plane, respectively. Figure 9 displays the ray path in a rotating meridian plane of the SM (Solar Magnetic) coordinate system when using the $\mathrm{k}$ vector orientation at the tilted pair derived above, i.e. $127^{\circ}$ in azimuth and $120^{\circ}$ in zenith angle. Successive positions of the wave energy are pointed along the ray path (a straight line in the physical 3-D space) at equal $\left(0.5 R_{\mathrm{E}}\right)$ distances from each other. We consider the meridian planes at each position, and fold all these onto a single plane, presented in Fig. 9. This plane is labelled $R-Z$, respectively for abscissa and ordinate, where $R$ is the distance of a point position from dipole axis and $Z$ its distance from the equatorial magnetic plane. The tilted pair is sketched as a red diamond in $R-Z$ plane. The ray path, shown by the blue crosses at successive positions of the backward trace, is twisted by the meridian projection, forming a curved line in the figure. $L$ shells of a dipolar magnetic field ( $L$ values 
from 3 to 11), intercepted by each meridian planes, are superposed in $R-Z$ plane due to the axial symmetry of the dipolar model. They are shown in black, as is the Earth's surface. IsoFce surfaces calculated from the dipole model of magnetic field, also presenting an axial symmetry, are plotted as green dotted lines. The isoFce surface corresponding to the df value fitting spectral properties of the continuum signatures $(d f=2.5 \mathrm{kHz})$ is plotted as a dashed red line. Following the approach of Gough (1982) mentioned above, we look for the source region of NTC emissions at the intersection of ray path with the isoFce surface at $d f=2.5 \mathrm{kHz}$. The directivity analysis via the DSM tool indicates two possible (and opposite) directions. An important clue for selecting one of the two is that the waves are emitted near local plasma frequencies within a density gradient (Grimald et al., 2008), in this case in the $\sim 15-25 \mathrm{kHz}$ frequency band. This eliminates the part of ray path directed toward high latitudes because plasma frequencies in the $15-25 \mathrm{kHz}$ band are infrequent in the polar cap region, especially under low geomagnetic activity. The ray path directed toward the equator, which is a better candidate, intersects the isoFce curve at $F_{\mathrm{ce}}=d f$ near equator. This provides a first order approximation of NTC source position $\left(R \sim 11 R_{\mathrm{E}}, Z \sim-0.5 R_{\mathrm{E}}\right)$.

The corresponding $L$ shell value, $L \sim 11$, is too high for the dipole model of magnetic field to be realistic, especially in the night MLT sector. This is the reason why successive positions along the ray path have been followed in a realistic model (Tsyganenko and Stern, 1996) tuned to the magnetic activity index of the day. Magnetic field lines from the realistic model, no longer in planes, and not presenting an axial symmetry anymore, are not shown in Fig. 9. As happens with the dipole model, the gyro-frequency encountered along the path toward the equator increases and then decreases. It decreases closer to the spacecraft than with the dipole model, though, and meets the required value $\left(F_{\mathrm{ce}}=2.5 \mathrm{kHz}\right)$ at a similar $L$ shell value, but at medium latitudes, far from the equator. The new estimated position of the radio source of NTC emissions $\left(X_{\mathrm{GSE}}=-1.2 R_{\mathrm{E}}\right.$, $Y_{\mathrm{GSE}}=-6.45 R_{\mathrm{E}}, Z_{\mathrm{GSE}}=-4.1 R_{\mathrm{E}}$ ) is indicated by a yellow star in Fig. 9, and the magnetic field vector at the source is indicated by an orange arrow. The source is placed in the dawn sector, $\mathrm{MLT}=06: 33$, at $5.3 R_{\mathrm{E}}$ from the tilted pair. The arbitrary chosen angular size of $20^{\circ}$ discussed above (end of Sect. 4.2) would thus correspond to a source diameter of about $2 R_{\mathrm{E}}$, a size with which the DSM tool can be safely used as defined (i.e. ignoring the source size). The position of NTC source region has been established via simple geometry, in particular under assumption of a linear ray path (free propagation), within a modelled magnetic field. The MLT sector obtained (dawn) is where NTC sources are expected (Gurnett, 1975; Grimald et al., 2007). The high value of $L$ shell, as well as the medium latitude, are less expected, and must be discussed in the context of the particular geophysical scenario prevailing for the 18 May 2008 event, which is done in Sect. 5.3 below. Prior to that, we estimate the precision of the position derived, and we compare the result with the position obtained from triangulation.

In order to evaluate the quality of the Tsyganenko model used, we have compared the magnetic field vectors measured onboard Cluster during NTC observations with vectors provided by the model. The comparison is excellent, indicating however a modelled magnetic field intensity slightly higher (of $\sim 5 \%$ ) than the measured value. The latter value is measured by two different instruments, the magnetometer (Balogh et al., 1997) and the sounder (Décréau et al., 1997), both instruments agreeing within $0.2 \%$. The discrepancy between measured and modelled values points out the inherent difficulty of using a modelled magnetic field in the 3-DisoFce approach, and leads to the following question: how sensitive is the source position to the prescribed $F_{\mathrm{ce}}$ value? In practice, a deviation of $5 \%$ of prescribed $F_{\text {ce }}$ value results, in all realistic models tested, in a displacement of the source of about $0.15 R_{\mathrm{E}}$, which is negligible when compared to uncertainties due to other factors controlling our evaluation of source position. The main source of uncertainty is the large dispersion of ray path orientation angles shown in Fig. 7. When applying the 3-D-isoFce method with inputs sweeping orientation values according to dispersion shown in Fig. 7, we obtain a region of source positions crudely within a sphere of $1 R_{\mathrm{E}}$ dimension. Finally, taking account of various other factors, as the uncertainty in the frequency distance between bands, $d f$, as well as ray path curvature within the outer plasmasphere, we can safely assume that the source is not placed near the equator, but at higher latitudes. The source region resulting from our uncertainty analysis is schematically represented by the orange oval displayed in Fig. 9.

We note that the source position derived from the 3-D-isoFce method $\left(X_{\mathrm{GSE}}=-1.2 R_{\mathrm{E}}, Y_{\mathrm{GSE}}=-6.45 R_{\mathrm{E}}\right.$, $\left.Z_{\mathrm{GSE}}=-4.1 R_{\mathrm{E}}\right)$ is far from the position found via triangulation and modulation factors $\left(X_{\mathrm{GSE}}=-9 R_{\mathrm{E}}, Y_{\mathrm{GSE}}=\right.$ $3.8 R_{\mathrm{E}}, Z_{\mathrm{GSE}} \sim-1.7 /-11.7 R_{\mathrm{E}}$ ). It is interesting to examine whether the inconsistency could be explained by our study (Sect. 3.3) about systematic errors in azimuth when ignoring the wave ellipticity. Figure 8 displays, in addition to the source position obtained from triangulation (ST), the position estimated by the 3-D-isoFce approach, indicated by a yellow star. Ray paths in $X-Y$ GSE plane, which we consider here as the "true" ones, joining each satellite $(\mathrm{C} 1$, the $\mathrm{C} 3-\mathrm{C} 4$ pair and C2) to the second source position (the star), are plotted as dashed lines. They make significant angles with directivity paths (solid lines) obtained under the first (triangulation) approach. In order to test our interpretation, we compare the three angle difference values $\delta, \delta=10^{\circ}$ from $\mathrm{C} 1$ viewpoint (black lines), $\delta=2^{\circ}$ from $\mathrm{C} 4$ viewpoint (blue lines), and $\delta=-10^{\circ}$ from $\mathrm{C} 2$ viewpoint (red lines), respectively, with the magnitude of errors in azimuth to be expected. As a work frame, we consider that the electric wave ellipse ratio is, when reaching $\mathrm{C} 2$ and $\mathrm{C} 1$, similar to what is measured by the tilted pair $(e \sim 0.85)$, and that the ray path 
inclination angles with spin planes are of the order $35^{\circ}$, a figure compatible with measured modulation ratios. In that context, the 3 error values can be translated, from curves in top right panel of Fig. 5, into $\lambda$ values evolving from $\sim 120^{\circ}$ at $\mathrm{C} 1$, to near $90^{\circ}$ at $\mathrm{C} 3 / \mathrm{C} 4$, then $\sim 60^{\circ}$ at $\mathrm{C} 2$. Such an evolution could be due to various curvatures of ray paths encountered along the first part of the source to satellite trajectory, before the radiation has reached the free space state. It could also be due to a Faraday rotation effect along the first part of the trajectory, before the wave has reached the so called "limiting polarization" discussed in Gurnett et al. (1988). It seems reasonable to consider a plasma thickness increasing from $\mathrm{C} 1$ (shorter distance, $4.82 R_{\mathrm{E}}$ ) to $\mathrm{C} 4$ (medium distance, $5.31 R_{\mathrm{E}}$ ) then $\mathrm{C} 2$ (similar distance, $5.33 R_{\mathrm{E}}$, with trajectory closer to Earth, thus meeting conditions of larger magnetization and density). Leaving a quantitative discussion to further studies, we can nevertheless check that the ellipse orientation at $\mathrm{C} 4$ position is consistent with this view. Indeed, thanks to the (tilted) additional satellite $\mathrm{C} 3$, we know all the characteristics of the electric field ellipse, including $\lambda$ value, at $\mathrm{C} 4$ position. This value is $\lambda=83^{\circ}$. The small positive error indicated by abacus of Fig. 5 for $\lambda=83^{\circ}$ is consistent with the error in azimuth found in Fig. $8\left(+2^{\circ}\right)$.

Finally, we examine the information given by the electric field magnitude observed on board $\mathrm{C} 1, \mathrm{C} 4$ and $\mathrm{C} 2$, the 3 spacecraft which share the same spin axis orientation. Figure $7 \mathrm{~d}$ indicates that electric field magnitudes are progressively decreasing, from $\mathrm{C} 1$ to $\mathrm{C} 4$ then $\mathrm{C} 2$, and that the magnitude ratios vary with the frequency considered. We consider the electric field magnitude integrated over the band chosen to derive directivity properties. This magnitude amounts to 52,46 and 38, respectively on $\mathrm{C} 1, \mathrm{C} 4$ and $\mathrm{C} 2$, in the chosen unit $\left(10^{-7} V_{\mathrm{rms}} \mathrm{Hz}^{-1 / 2}\right)$. The first parameter which should control the measured wave magnitude is the distance from the source to the observing satellite. Within the frame of a spherical wave radiating homogeneously inside a given cone angle, electric field magnitudes are expected to vary as the inverse of these distances $d$ (electric field power varying as $d^{-2}$ ). The magnitude ratio from $\mathrm{C} 1$ to $\mathrm{C} 4,1.13$, is indeed comparable to the inverse of distances to source ratios, 1.10 (accounting for distances of 4.82 and $5.31 R_{\mathrm{E}}$, respectively), at least within a possible small gain mismatch resulting from uncertainty about instrument performances. However, the magnitude ratio from $\mathrm{C} 4$ to $\mathrm{C} 2,1.21$, cannot be explained by the inverse of relative distances, which is close to 1 . We note in addition (see Sect. 5.1) that the measured modulation factors are identical $(0.50)$ on $\mathrm{C} 2$ and $\mathrm{C} 4$, indicating that the inclination of ray paths with respect to spin planes (hence of electric field ellipses with spin plane) are similar on $\mathrm{C} 2$ and $\mathrm{C} 4$. We are left with questions related to possible interpretations. Is $\mathrm{C} 4$ placed nearer the centre of a cone angle issued from the source than $\mathrm{C} 2$ ? Is the visibility of the entire source surface better from $\mathrm{C} 4$ than from $\mathrm{C} 2$ ? Is the medium less transparent along the propagation path from the source to $\mathrm{C} 2$ than from the source to $\mathrm{C} 4$ ?

\subsection{General context and suggested scenario}

In this section, we confront WHISPER observations during the 18 May 2008 event (including derived position of NTC radio source) with the general geophysical context prevailing that day, and present a simple scenario explaining this. Specifically, we assume that the NTC source region we have derived is co-located with a plasmapause density gradient, and we discuss the overall shape of the plasmasphere in this situation, taking account of current knowledge about plasmasphere topology and dynamics. Two main considerations guide us: timing and morphology.

\subsubsection{Timing}

The time evolution of geophysical conditions over the few hours preceding the event, and during the event, is summarized in Fig. 10. Solar wind conditions shown in panels (a) to (e) are, successively, the magnetic field intensity $|B|$, the magnetic component $B_{Z}$ in GSE coordinate system, the solar wind velocity $V_{\mathrm{s}}$, the proton density $N_{\mathrm{p}}$ and the auroral index AE. Values measured in the solar wind are delayed in time, according to propagation down to the Earth's bow shock nose (NASA GSFC and CDAWeb data provided by J. H. King and N. Papatashvilli). The frequency-time spectrogram of the electric field intensity measured on board $\mathrm{C} 2$ is shown in panel (f). A dashed purple line drawn at $\sim 11: 10$ UTC points out that the start of low frequency AKR emissions $(50-80 \mathrm{kHz})$ observed on $\mathrm{C} 2$ spectrogram coincides with a sharp increase of the AE index. This auroral activity is probably triggered by an abrupt change in orientation of interplanetary magnetic field, $B_{z}$ turning positive (panel b).

The start of the studied NTC event occurs shortly after that, near a peak in the AE index variation. As in Fig. 6, the start time chosen for reference (11:50 UTC) is marked in Fig. 10 by a vertical solid line in purple. The overall spectral evolution of NTC waves with time (see also oval shape in Fig. 6c), bears some resemblance with the time evolution of the type referred to as the continuum enhancement, which supported Gough's isoFce paradigm (Gough, 1982). Such events have been observed and studied following Gough's study (Kasaba et al., 1998; Décréau et al., 2004). The spectral signature of the continuum enhancement is characterized by banded features, with frequencies globally increasing over time intervals of one to several hours, reported to develop during substorms, after the start of an electron injection event. Most NTC enhancement events reported so far display characteristics that can be compared to what is observed here. According to Kasaba et al. (1998), who analysed a significant number of such events observed from GEOTAIL satellite (48 events during years 1994-1995), the radiation frequency mainly ranged from 10 to $80 \mathrm{kHz}$, thus including the frequencies encountered on 18 May 2008. During this specific event, however, while the central frequency of the event increased, the frequency distance between bands 
a)

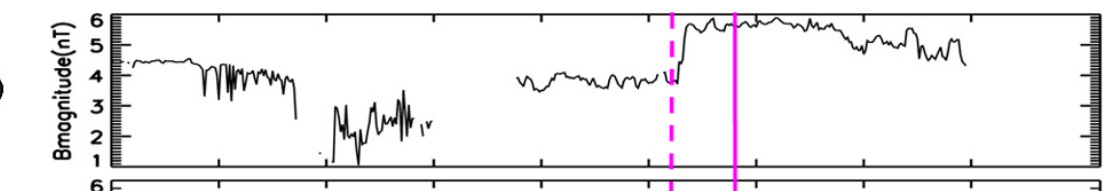

b)

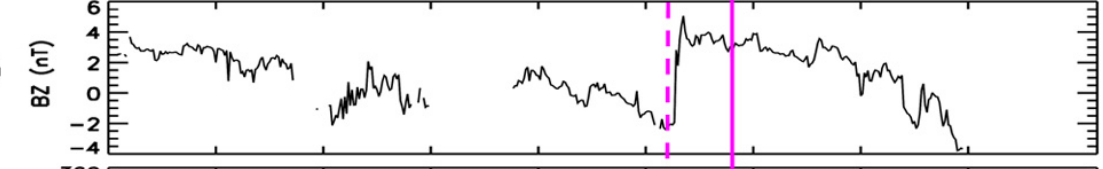

c)

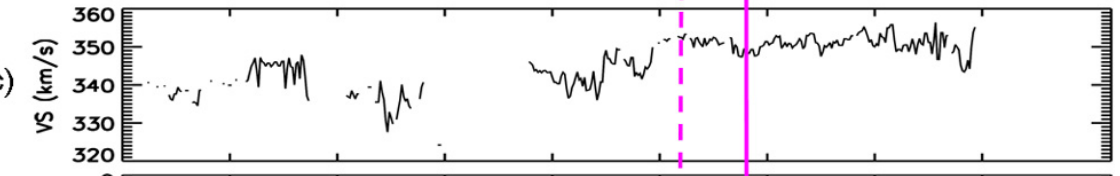

d)

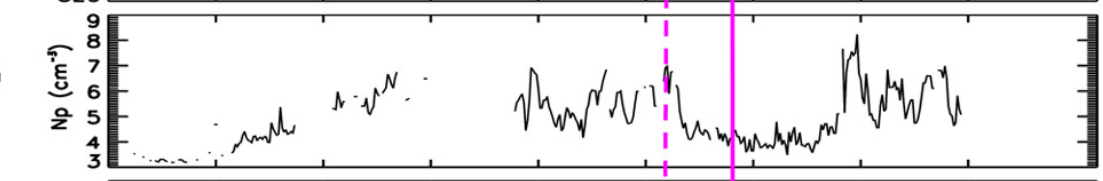

e)
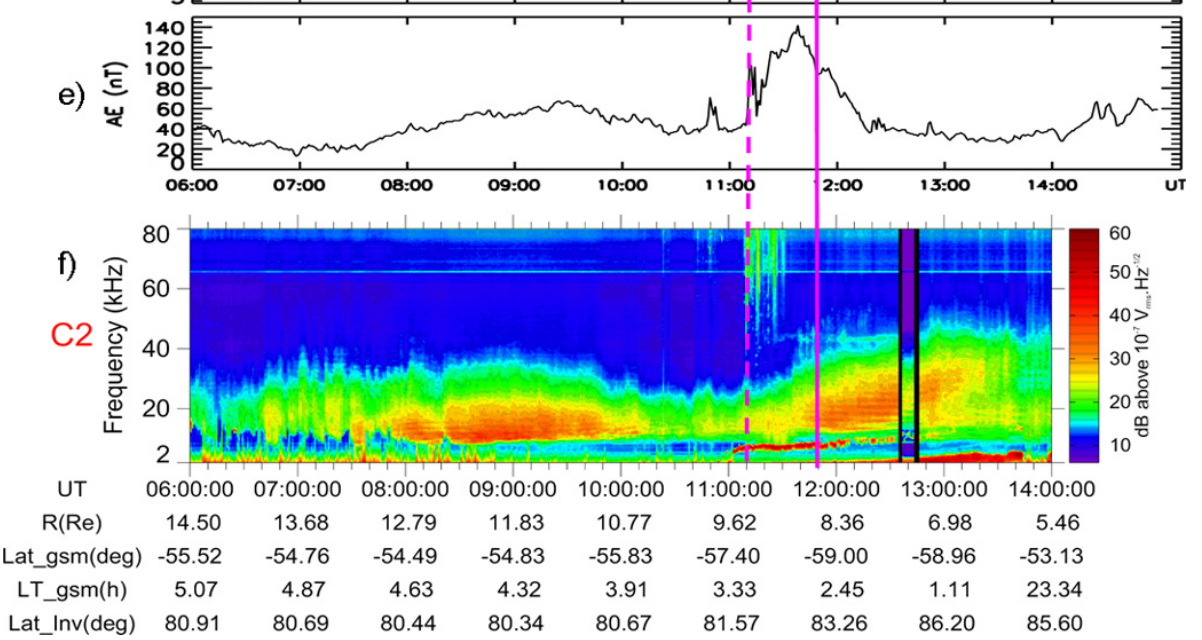

Fig. 10. Evolution of interplanetary and auroral parameters (panels a-e), and of radio emissions observed by WHISPER on board C2 (panel f), on 18 May 2008, from 06:00 to 14:00 UTC. A dashed line (at 11:10 UTC) points to a change in geophysical conditions. A solid line (at $\sim$ 11:50 UTC) indicates the start time of prominent NTC emissions studied (Fig. 6). According to the global shape of the spectral signature displayed, they belong to a NTC enhancement (see text). The apparent anomaly during the interval 12:37-12:43 UTC (sudden lowering of global intensity) is due to using the $E_{z}$ dipole antenna instead of the $E_{y}$ dipole antenna. The $E_{z}$ dipole having lost a sensor, its effective length has been divided by a factor 2 .

remained about stable, in contrast with most events reported so far. Near 12:50 UTC, the solar wind density and temperature increased, while the banded NTC structure moved to higher frequencies, and the frequency distance between bands increased slightly. At 13:00 UTC, the orientation of interplanetary magnetic field changed. Finally, NTC emissions resumed after 13:30 UTC.

A question to settle is whether the spectral characteristics of the NTC event, as observed by Cluster, reflect variations of source characteristics with time, or if they are due to a space effect (evolution linked to moving viewpoints). The Cluster constellation, placed near perigee, covers a significant distance during the development of the event (about $1 R_{\mathrm{E}}$ in $35 \mathrm{~min}$ ), thus providing a viewpoint over the source which is changing along the orbit. This could explain, at least partly, the evolution with time of the observed spectrograms (satellites entering then leaving a radio wave beam). However, all four spacecraft observe the same instantaneous frequency signature (Fig. 6d), despite the fact that inter-satellite distances are significant (larger than $1 R_{\mathrm{E}}$ for the largest distance). Moreover, they all display the same overall spectral evolution with time, with same start and stop times (at respectively $\sim 11: 40$ and 13:00 UTC) for C1 and C2 than on the tilted pair C3-C4 (figure not shown). We conclude that the observed time evolution of the event is due to a general time evolution of the magnetosphere, and not to a change in position of Cluster observatories. 

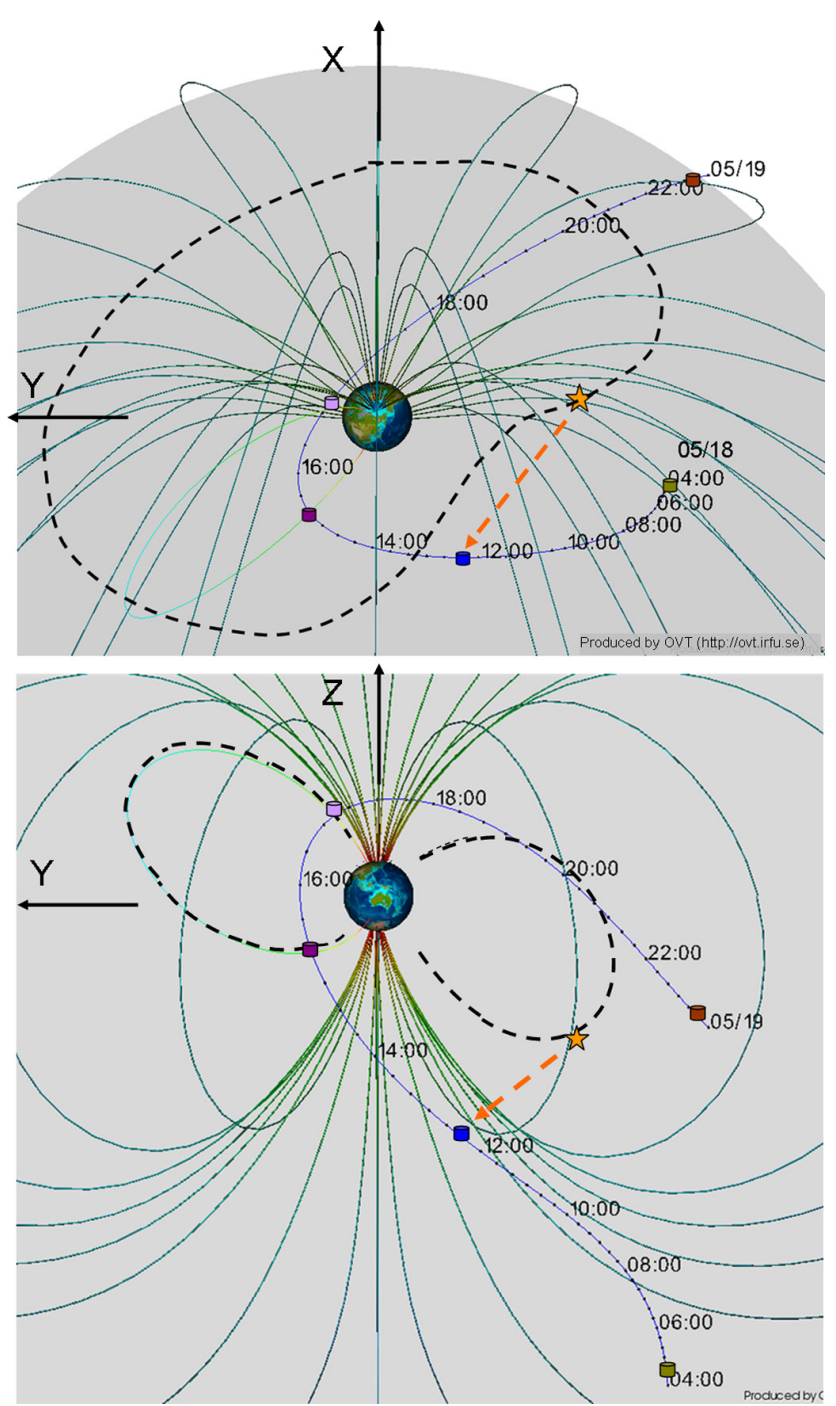

Fig. 11. Views in GSM coordinate system from OVT (Orbit Visualization Tool, http://ovt.irfu.se). C3-C4 orbit on 18 May 2008, and magnetopause field lines are projected unto $X-Y$ plane (top) and $Y-Z$ plane (bottom). The NTC source is indicated by a star; the observing point at 12:20 UTC by a blue symbol; other positions of the (C3-C4) pair by symbols in various colours. Plasmapause crossings, occurring at 15:20 UTC and 16:50 UTC in the dusk sector, are shown in dark and light purple. Magnetopause crossings, occurring at 04:20 UTC and 23:30 UTC, are shown in light green and brown. Dashed curves sketch possible positions of plasmapause, equatorial boundary in upper panel, selected magnetic flux tubes along plasmapause surface in lower panel.

\subsubsection{Morphology}

Concerning morphology, we have at our disposal factual knowledge of positions of two magnetopause crossings observed by Cluster/WHISPER on 18 May 2008, at respectively 04:20 UTC (inbound orbit element) and 23:30 UTC (outbound orbit element), as well as two plasmapause crossings (plasma frequency stepping from $\sim 20$ to $30 \mathrm{kHz}$ ) observed by the same instrument, at respectively 15:20 UTC and 16:50 UTC. Another piece of information, more conjectural as it is deduced from remote observations, is a third plasmapause position, more precisely a radiating part of plasmapause boundary layer forming the source region of NTC waves. Finally, we know that the ray path between the NTC source region and observing spacecraft (at 12:20 UTC) is free of high densities. The five positions of Cluster C4 at times quoted above (entering magnetosphere, observing NTC waves, entering plasmasphere, leaving plasmasphere, exiting magnetosphere), as well as the NTC source position, are shown in Fig. 11, which displays Cluster orbits and magnetopause surface in GSM coordinate system given by the OVT tool (http://ovt.irfu.se). Magnetic field lines along the magnetopause are projected onto the two views presented ( $X-Y$ plane and $Y-Z$ plane). The magnetic field flux tube of the first plasmapause layer crossed by $\mathrm{C} 4$ in the dusk sector is also shown (magnetic field line in light blue). It happens that the position of $\mathrm{C} 4$ on its second plasmapause crossing is close to this specific field line.

The positions of boundaries listed above are consistent with the available information about geophysical activity for this event. The observed position of the magnetopause is where models (Boardsen et al., 2000), and statistics (Dušík et al., 2010) predict it should be under solar wind conditions prevailing that day. This magnetopause boundary is far from the found position of the NTC source region: it is placed, at its closest distance, at $1.8 R_{\mathrm{E}},-10.6 R_{\mathrm{E}}$ and $-7.5 R_{\mathrm{E}}$ from the source, respectively along $X, Y$ and $Z$ axis in GSM coordinate system (Tsyganenko model of 2004). Such a large distance excludes a NTC source region placed at the magnetopause gradient. Concerning the plasmasphere body it is expected, owing to the exceptionally quiet magnetic activity conditions during hours preceding the 18 May 2008 event (see am geomagnetic indices in Fig. 6a), that this region is expanded to large distances (see, for instance, Fig. 1 in Carpenter and Anderson, 1992). This is indeed what happens that day: the first encounter by Cluster of the plasmapause boundary layer occurs at a large $L$ shell value $(L=8.1)$, when $\mathrm{C} 3$ and $\mathrm{C} 4$ are travelling at medium magnetic latitudes (Mlat $\sim-54^{\circ}$ ) in the night sector $(\mathrm{GSM} \mathrm{LT}=21.7)$. A couple of hours later (16:48 UTC), the C3-C4 pair leaves the plasmasphere, crossing approximately the same magnetic field tube $(L \sim 8.5)$ near the opposite magnetic footprint $\left(\right.$ Mlat $\left.\sim 45^{\circ}\right)$ in the day sector $($ GSM LT $=16.7)$.

The plasmasphere, greatly expanded after the NTC wave episode, at least inside dusk sector, could have been expanded at still higher $L$ shells before and during the observed emission in the dawn sector and closer to equator. We could, unfortunately, not find suitable in situ data in the local time sector of the NTC source for locating the plasmasphere boundary that day, nor for checking the presence of electron injection at medium energy, which could have triggered the emission. It is also difficult to use the ray path orientation as 
an indicator of the plasmapause surface orientation near the source. The beaming theory elaborated by Jones (1982) indicates that the angle between the plasmapause gradient and the emitted ray path can be derived from the ratio between the plasma frequency $F_{\mathrm{p}}$ and the gyrofrequency $F_{\mathrm{ce}}$ in the source region. This theory, which in this case would give a small angle of about $20^{\circ}$, has not been confirmed in practice, because the model (a single point source) and a suitable observatory (crossing two beams of small angular size, issued from the same source) are not met in reality (Morgan and Gurnett, 1991; Grimald et al., 2007). Observations from Green et al. (2002) suggest that a density notch formed in the plasmasphere body can focus radio emissions along a beam of reduced angular size. Both approaches suggest a beam radiating along a direction not far from normal to the main, large scale, plasmapause surface. In order to fit this scenario with the observed ray path orientation in the $X-Y$ plane (toward the night-dusk sector), we propose that a shoulder could have been formed at the start of the active period, together with particle injections triggering the NTC radiation. Shoulders are indeed known to form in the dawn sector (Pierrard and Lemaire, 2004). The ray path orientation in the $Y-Z$ plane (toward south) would fit emission from medium latitudes of a plasmapause $L$ shell surface elongated near the equator. Figure 11 displays a crude sketch of a plasmapause boundary layer (black dashed curves) in agreement with the morphology described above. This boundary is, in the upper panel, shown as its equatorial extension. In the bottom panel, we have figured the magnetic field line at the plasmapause crossed by Cluster in the dusk sector, as well as the magnetic field line in the dawn sector associated with the NTC region source. Such a sketch can only be an oversimplified representation of the actual expansion of the plasmasphere body. It suggests a toroidal shape of the plasmasphere, with a smooth "byte out", i.e. magnetic flux tubes entirely deprived of plasmaspheric material, in the 00:00-06:00 MLT sector. A more complex structure of the large scale plasmapause surface could also fit the observed ray path orientation. Actually, at large $L$ values and under quiet magnetic conditions, the corotating magnetic field tubes are only partially replenished by ionospheric sources (Lointier et al., 2013). This could lead to complex 3-D shapes of the plasmasphere boundary, in particular during a surge of activity, when plasma irregularities can be possibly eroded from the plasmasphere (Darrouzet et al., 2004).

\section{Summary and conclusion}

This paper presents a detailed study of a NTC wave event observed during the tilt campaign run by the Cluster mission. The study yields three main results:

1. the validation of a novel experimental concept, where two spacecraft are associated for measuring electromagnetic waves;
2. the measurement of 3-D geometric characteristics of electric field, opening a source localization approach forbidden to a large variety of NTC observations;

3. the best estimation of the position of NTC radio waves sources for the studied event and a proposed scenario within the frame of geophysical conditions prevailing that day.

The experimental system that had to be validated combines two long dipolar electric antennas carried by the two satellites $\mathrm{C} 3$ and $\mathrm{C} 4$, close enough to each other to be considered as placed at a single point in space. We show that this system, where antennas rotate in two planes at an angle from each other, is capable of measuring the 3-D characteristics of propagating plane waves, in particular the shape of electric field ellipse, and the direction of vector normal to the wave plane. We present the software tool which has been designed to meet the specific experimental constraints encountered, wave instrumentation (WHISPER experiment), as well as spacecraft configuration (tilted Cluster pair) and equipment (onboard data handling), under the hypothesis of a point source. We show that this tool, when applied to experimental data, leads to a satisfying solution in most frequency-time domains for the event treated, which is a first indication that the measured electric field behaves as expected. The performances achieved in the case of our selected event are limited by fluctuations in the observed NTC waves. An analysis based on statistics leads to a variance of $\pm 10 \%$ in ellipse ratio, of $\pm 2.5^{\circ}$ in zenith angle and of $\pm 5^{\circ}$ in azimuth. The limited instrument performances, in particular the time resolution, do not allow examination of what causes the observed dispersion in electric field geometry, which could be due to fluctuations at the source, during propagation, or at reception. When observed waves are stemming from several sources, or from an extended source, the modulation parameters measured are smaller than in the case of a single point source. Sources of large extension lead to an important squeezing of the modulation parameters. In such a case, the DSM tool does not provide any solution to ellipse characterization (as observed during the tilt campaign when dealing with $2 F_{\mathrm{p}}$ radiation events). Within the frame of a source of limited extension, as we think is the case here, we expect that the angular error on the wave vector orientation depends on the actual geometry encountered (orientation of polarization ellipse with respect to the tilted pair, source properties), as has been demonstrated by Cecconi (2007) in the field of goniopolarimetry. We leave to a future work such a study applied to a tilted pair device.

The main benefit of the full 3-D characterization of electric field geometry is the capacity to estimate ray path orientation in 3-D at the observatory. The source localization can then be done via the "3-D-isoFce" method, which combines simple ray tracing and knowledge of magnetic field magnitude at the source (estimated from spectral signatures). The source position found is in agreement with the magnitude 
ratios measured from two spacecraft, $\mathrm{C} 1$ and $\mathrm{C} 4$, placed at different distance from the source. The importance of the progress to be expected from a tilted satellite pair device, when compared to a single satellite using the "spin - null" method, is illustrated by two ancillary results.

The first result is derived from the quantitative study of systematic errors due to use of the circular polarization assumption and is presented in the form of graphics (Fig. 5). Those graphics show that, even in the case of quasi-circular polarization (illustrated by a value of ellipticity ratio equal to 0.85 ), systematic errors on determination of azimuths can be important. As a consequence, the measured modulation factor values cannot be a secure means to estimate ray path elevation angle over spin plane. None of those results are really surprising, but this paper provides quantitative information of use for interpretation of spin modulation analysis of NTC radio wave observations.

The second result is the obvious failure of the triangulation method (using 2-D directivity via classical spin modulation) to point to the correct source position. This can be explained by systematic errors in directivity angles derived via spin modulation when interpreted within the frame of fully circular polarisation. Actually, although modest (a few degrees), systematic errors can be different for waves observed from one of the observing spacecraft and for waves observed from another spacecraft. A possible interpretation is that systematic errors evolve gradually from one observing point to another. This specific behaviour could have been overlooked without the opportunity of comparison between the two approaches offered by the Cluster tilt campaign.

The position of the source region (dawn sector, at large distance both from Earth and the magnetopause, at medium latitudes along a plasmapause boundary) is compatible with the observed conditions of geomagnetic activity. One interesting feature is the orientation of ray path, directed from the flank of the plasmasphere toward the polar cap. This finding does not allow deriving the shape and dimension of the source region, but the orientation of ray path points out the importance of a 3-D geometry of plasmapause layer, likely associated to density irregularities of large dimension. Global images have revealed the complexity of plasmasphere topology and dynamics but they are limited to density values above about $40 \mathrm{~cm}^{-3}$ (Goldstein et al., 2007), associated with plasma frequencies higher than those considered here. Local multipoint observations made by Cluster (Darrouzet et al., 2009), as well as statistical views about the expanded plasmasphere (Lointer et al., 2013), which have been pursued in recently explored regions, could also shed light about morphology of highly expanded plasmaspheric regions.
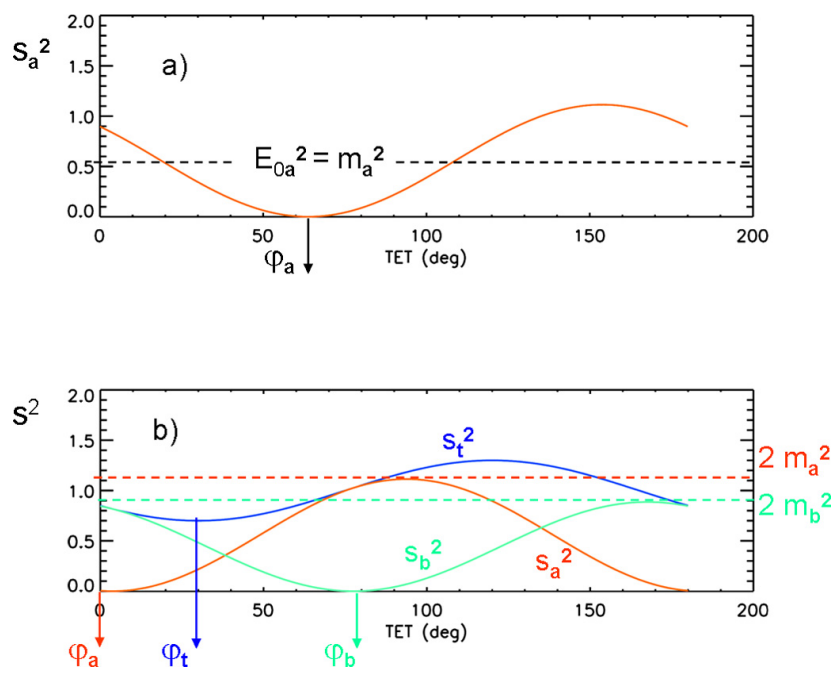

Fig. A1. Variation of measured signal (electric field power density) as a function of antenna attitude $\theta$ in case of (a) linear polarization; and (b) elliptic polarization, where the measured signal $s_{\mathrm{t}}^{2}$ is the sum of two linear components $s_{a}^{2}$ and $s_{b}^{2}$, projected in spin plane.

\section{Appendix A}

\section{DSM tool algorithm}

\section{A1 Case of linear polarization}

In the case of linear polarization, recognized by a fully modulated signal (Fig. A1a) on both satellites, the wave plane cannot be determined. The DSM algorithm calculates the characteristics of the linear wave component, a $\boldsymbol{u}$. The positions of meridian planes $P_{4}$ and $P_{3}$ are deduced from the measured parameters $\varphi_{4 \mathrm{t}}$ and $\varphi_{3 \mathrm{t}}$, attitude angles where the received signal on $\mathrm{C} 4$ and $\mathrm{C} 3$ is null. The two planes are intersected in order to derive the components of the vector $\boldsymbol{u}$. The magnitude a can be estimated as $a_{4}=2 E_{04} / \sin \sigma_{4}$, or as $a_{3}=2 E_{03} / \sin \sigma_{3}$, where $\sigma_{4}$ and $\sigma_{3}$ are the zenith angles of $\boldsymbol{u}$ in [Sc4] and [Sc3] coordinate systems, respectively, and $E_{04}$ and $E_{03}$ the magnitudes of the measured signals on C4 and $\mathrm{C} 3$ respectively. The distance between both estimations is measured by the following factor:

$d a=\frac{\left|a_{3}^{2}-a_{4}^{2}\right|}{\left(a_{3}^{2}+a_{4}^{2}\right)}$

which belongs to the interval 0 to 1 , and should be equal to 0 if measurements are free of noise and other limits.

\section{A2 Case of elliptic polarization}

\section{A2.1 Measured characteristics of the modulated signal expressed as the sum of two sine components}

The two sine functions, $s_{a}^{2}$ and $s_{b}^{2}$, of the same periodic variable $\theta$ correspond to the contributions in a spin plane of the 
two linear waves forming the elliptical wave. Their sum, $s_{\mathrm{t}}^{2}$ (blue curve in Fig. A1b), is expressed as a sine function of $\theta$. Four input quantities, $m_{a}^{2}, \phi_{a}, m_{b}^{2}$; and $\phi_{b}$, are needed to define the two contributions, and three output quantities, $E_{0}^{2}$, $\alpha$ and $\phi_{\mathrm{t}}$, are needed to describe their sum. The relations between input and output quantities are obtained by writing that the identity $s_{a}^{2}+s_{b}^{2} \equiv s_{\mathrm{t}}^{2}$ must be satisfied at all values of the common variable, $\theta$ :

$$
\begin{aligned}
& m_{a}^{2}\left[1-\cos 2\left(\theta-\varphi_{a}\right)\right]+m_{b}^{2}\left[1-\cos 2\left(\theta-\varphi_{b}\right)\right] \\
& \quad \equiv E_{0}^{2}\left[1-\alpha \cos 2\left(\theta-\varphi_{\mathrm{t}}\right)\right] .
\end{aligned}
$$

Identity of continuous (un-modulated) components leads to

$m_{a}^{2}+m_{b}^{2}=E_{0}^{2}$.

Let us define $r$, ratio of wave intensities, as

$r=m_{b}^{2} / m_{a}^{2} \quad$ or $\quad r=m_{a}^{2} / m_{b}^{2}, \quad$ such that $r \leq 1$.

Identity of modulated components can be written as

$\cos 2\left(\theta-\phi_{a}\right)+r \cos 2\left(\theta-\phi_{b}\right) \equiv \alpha(1+r) \cos 2\left(\theta-\phi_{\mathrm{t}}\right)$.

Let us introduce $\zeta$ and $\delta$ as

$\left.\zeta=2\left(\phi_{a}-\phi_{b}\right)\right)$

and

$\delta=2\left(\phi_{a}-\phi_{\mathrm{t}}\right)$

and define $\gamma$ by

$\tan \gamma=\frac{r \sin \zeta}{1+r \cos \zeta}$.

As a consequence of the symmetry of antenna (sensors placed on each tip of the antenna having the same efficiency, and the antenna measuring only the power and not the phase of the signal), we do not lose the generality of the problem by restricting the domain of variation of angles $\theta, \phi_{a}, \phi_{b}$ and $\phi_{\mathrm{t}}$ to $0, \pi$, and of angles $\zeta$ and $\delta$ to $0,2 \pi$, or, equivalently, to $-\pi, \pi$. As concerns the angle $\gamma$, it can be chosen in the domain $-\pi / 2, \pi / 2$.

Equation (A5) writes

$\frac{1+r \cos \zeta}{\cos \gamma} \cos \left[2\left(\theta-\varphi_{a}\right)+\gamma\right] \equiv \alpha(1+r) \cos \left[2\left(\theta-\varphi_{a}\right)+\delta\right]$,

where coefficients of cosine functions are both positive. This leads to

$\gamma=\delta$.

Identical magnitudes in Eq. (A9) lead to

$\frac{1+r \cos \zeta}{\cos \gamma}=\alpha(1+r)$
Combination of Eqs. (A8) and (A11) allows eliminating the parameter $\gamma$, and leads to

$x=\frac{\alpha^{2}(1+r)^{2}-\left(1+r^{2}\right)}{2 r}$, where $x=\cos \zeta$.

In summary, the square mean power $E_{0}^{2}$ is derived from Eq. (A3), the angle $\phi_{\mathrm{t}}$ from Eq. (A7), combined with Eqs. (A10), (A8) and (A4), and the modulation index $\alpha$ from Eq. (A11).

\section{A2.2 Exploration of the domain of input combinations leading to the measured modulation characteristics}

Knowing the modulation parameters measured from a given platform (C4 or $\mathrm{C} 3)$, one explores all possible inputs (linear components projected in spin plane) that lead to the given observation. To do this, we can use any one of the free parameters, $r, x, \zeta$ or $\delta$, linked by Eqs. (A10) to (A12). The parameter $x$ varies when $\mathrm{r}$ decreases from 1 toward 0 between the limits $2 \alpha^{2}-1$ (at $r=1$ ) and -1 (as $\zeta$ must be real). This defines two possible $\zeta$ angle intervals, $\left[\operatorname{acos}\left(2 \alpha^{2}-1\right), \pi\right]$ and $\left[-\pi,-\operatorname{acos}\left(2 \alpha^{2}-1\right)\right]$, depending on sign of $\zeta$, which can be summarized as $|\zeta|$ belonging to interval $\left[\operatorname{acos}\left(2 \alpha^{2}-1\right), \pi\right]$.

We choose to explore the domain of input combinations by sweeping the $|\zeta|$ angle interval in small steps (typically, $\left.d|\zeta|=1^{\circ}\right)$. The $r$ value is uniquely derived from each value of $|\zeta|$ by Eq. (A12) and condition $r \leq 1$. One gets

$r=-\frac{\left(x-\alpha^{2}\right)}{1-\alpha^{2}}-\sqrt{\frac{\left(x-\alpha^{2}\right)^{2}}{\left(1-\alpha^{2}\right)^{2}}-1}$

and Eq. (A11) coupled to Eq. (A10) leads to

$\cos \gamma=\frac{1+r x}{\alpha(1+r)}$.

One can check that the right side of Eq. (A14) is smaller than unity. The angle $\gamma$ (or $\delta$ ) leads to $\phi_{a}$, then $\phi_{b} ; E_{0}^{2}$ and $r$ lead to $m_{a}^{2}$ and $m_{b}^{2}$.

As an illustration, we take the example of the measurements shown in Fig. 1. The modulation index on $\mathrm{C} 4$ is $\alpha=0.502$. The angle $|\zeta|$ varies in the domain $[120-180]^{\circ}$, which is explored in 61 steps. Two intervals for angle $\zeta$ are explored: -180 to $-120^{\circ}$ and 120 to $180^{\circ}$. The modulation index on $\mathrm{C} 3$ is $\alpha=0.191$. The associated angle $|\zeta|$ (measured in coordinate system $\left[S_{\mathrm{C} 3}\right]$ ) varies in the domain [158$180]^{\circ}$, which is explored in 23 steps of $1^{\circ}$. Two intervals are explored: -180 to $-158^{\circ}$ and 158 to $180^{\circ}$.

\section{A2.3 The DSM tool algorithm}

Preliminary to any further treatment, we test if $\mathrm{C} 3$ and $\mathrm{C} 4$ signals at phase zero are equal (within $\pm 20 \%$ ), rejecting all cases where it is not the case. We also check the eventuality of a purely linear polarization, and treat it as indicated 
above (Sect. A1). The case of a purely circular polarization can be recognized by the fact that both spin planes cut the polarization circle along a diameter. As a consequence, both received signals peak at the same intensity (see illustration in Fig. 4). The DSM tool algorithm does not check this eventuality specifically.

In the general case, the DSM algorithm follows a strategy in six steps.

1. The $|\zeta|$ angle interval, $\left[\operatorname{acos}\left(2 \alpha^{2}-1\right), \pi\right]$, is explored in steps of $1^{\circ}$. Equations (A10) to (A12) provide corresponding values of $x, r$ and $|\delta|$. Noting from Eqs. (A9) and (A10) that $\delta$ and $\zeta$ have the same sign, two solutions for the pair $\phi_{a}$ and $\phi_{b}$ are derived according to Eq. (A7), the pair $\varphi_{a}=\varphi_{\mathrm{t}}+\frac{|\delta|}{2}$ and $\varphi_{b}=\varphi_{a}-\frac{|\zeta|}{2}(\zeta$ and $\delta>0)$ on one hand, the pair $\varphi_{a}=\varphi_{\mathrm{t}}-\frac{|\delta|}{2}$ and $\varphi_{b}=$ $\varphi_{a}+\frac{|\zeta|}{2}$ on the other hand, with $\zeta$ and $\delta<0$. Related quantities $m_{a}^{2}$ and $m_{b}^{2}$ are calculated from Eqs. (A3) and (A4). This step provides a series of N3 quadruplets $\left(\varphi_{a}, \varphi_{b}, m_{a}, m_{b}\right)_{3}$ from $\mathrm{C} 3$ observations, and a series of N4 quadruplets $\left(\varphi_{a}, \varphi_{b}, m_{a}, m_{b}\right)_{4}$ from C4 observations. In the chosen example, $N 3=45$ and $N 4=121$.

2. All possible instances of quadruplets are combined in order to derive, for each combination, a set of $\boldsymbol{u}, \boldsymbol{v}, a$ and $b$ values where $a \boldsymbol{u}$ and $b \boldsymbol{v}$ are candidates of linear electric field components. Vector $\boldsymbol{u}$ is derived from cross product of unit vectors normal to meridian planes $P_{4 a}$ and $P_{3 a}$, written as

$I_{4}=\left[\begin{array}{c}\cos \left(\varphi_{a 4}\right) \\ \sin \left(\varphi_{a 4}\right) \\ 0\end{array}\right]$ and $I_{3}=\mathbf{M}^{-1} \times\left[\begin{array}{c}\cos \left(\varphi_{a 3}\right) \\ \sin \left(\varphi_{a 3}\right) \\ 0\end{array}\right]$,

where $\mathbf{M}$ is the transformation matrix from $\left[S_{\mathrm{C} 3}\right]$ coordinate system to $\left[S_{\mathrm{C} 4}\right]$ system. Vector $\boldsymbol{v}$ is derived similarly, from $\phi_{b 4}$ and $\phi_{b 3}$ of chosen quadruplet combination.

The magnitudes $\mathrm{a}$ and $\mathrm{b}$, measured from $\mathrm{C} 4$ platform, are given by $a_{4}^{2}=\frac{4 m_{a 4}^{2}}{\left(u_{x}^{2}+u_{y}^{2}\right)}$ and $b_{4}^{2}=\frac{4 m_{b 4}^{2}}{\left(v_{x}^{2}+v_{y}^{2}\right)}$, where $u_{x}$ and $u_{y}$, respectively $v_{x}$ and $v_{y}$, are components of $\boldsymbol{u}$ and $\boldsymbol{v}$ in $\mathrm{C} 4$ spin plane. Expressions of $\boldsymbol{u}$ and $\boldsymbol{v}$ are derived in $\left[S_{\mathrm{C} 3}\right]$, which lead, similarly, to magnitudes $a_{3}$ and $b_{3}$ measured from $\mathrm{C} 3$ spin plane.

3. A selection procedure allows to reach an acceptable solution, i.e. a solution which satisfies the model: $\boldsymbol{u}$ and $\boldsymbol{v}$ vectors at right angle, same magnitudes as measured from each satellite. Having combined N3 and $\mathrm{N} 4$ series of quadruplets, one obtains $2 \cdot \mathrm{N} 3 \cdot \mathrm{N} 4$ candidate pairs $\left(\boldsymbol{u}_{\mathrm{c}}, \boldsymbol{v}_{\mathrm{c}}\right)$, and associated magnitude values: (i) $a$, via estimations $a_{3}$ and $a_{4}$, and (ii) $b$, via estimations $b_{3}$ and $b_{4}$. For every pair, one calculates three quantities, which should optimally be equal to zero: $d a=\frac{\left|a_{3}^{2}-a_{4}^{2}\right|}{\left(a_{3}^{2}+a_{4}^{2}\right)}, d b=\frac{\left|b_{3}^{2}-b_{4}^{2}\right|}{\left(b_{3}^{2}+b_{4}^{2}\right)}, p_{u v}=|u . v|$. Their sum $\Delta=d a+d b+p_{u v}$ is also derived. The selection of the optimal solution is made in two steps: first eliminate all occurrences where $d a, d b$ and $p_{u v}$ are larger than thresholds fixed by measurements uncertainties (typically 0.05 or 0.1 ); and then, among selected occurrences (which provide similar solutions), selecting, empirically, the one with smaller $\Delta$. When the first step does not succeed, then the model of elliptical electric field is declared non-valid.

4. A final solution $(\boldsymbol{u}, \boldsymbol{v}, \boldsymbol{k}, a, b)$ is derived from the best acceptable set of parameters obtained after the selection procedure, say $\boldsymbol{u}_{\mathrm{c}}, \boldsymbol{v}_{\mathrm{c}}, \boldsymbol{k}_{\mathrm{c}}=\boldsymbol{u}_{\mathrm{c}} \hat{\boldsymbol{v}}_{\mathrm{c}}, a_{3}, b_{3}, a_{4}$, and $b_{4}$. The unity vectors $\boldsymbol{u}$ and $\boldsymbol{k}$ are simply $\boldsymbol{u}_{\mathrm{c}}$ and $\boldsymbol{k}_{\mathrm{c}}, \boldsymbol{v}$ completing the orthogonal base. Electric field magnitudes $a$ and $b$ are derived from the averaged intensities $a^{2}=1 / 2\left(a_{3}^{2}+a_{4}^{2}\right)$ and $b^{2}=1 / 2\left(b_{3}^{2}+b_{4}^{2}\right)$.

5. Vectors $\boldsymbol{u}, \boldsymbol{v}, \boldsymbol{k}$ are expressed in GSE coordinate system.

6. The algorithm derives a solution responding to geometrical considerations expected in the case of a circular polarization. Coordinates of $\boldsymbol{k}$ vector are calculated by intersecting the meridian planes containing spin axes and receiving antenna at minimum signal, respectively on $\mathrm{C} 3$ and on C4 (Fig. $3 b$ in Sect. 2.2). The quality of this solution is estimated from a parameter $\Delta_{\text {circ }}$ comparable to the parameter $\Delta=d a+d b+p_{u v}$ defined above. Our approach leads to $p_{u v}=0$ (the major and minor axis of the "ellipse" are normal to each other) and to $d a=d b$ (major and minor axis are equal). The quantity $d a$ is derived in comparing the maximal electric power magnitudes, respectively on $\mathrm{C} 3$ and $\mathrm{C} 4$, i.e. $d a=\frac{\left|s_{\max 3}^{2}-s_{\max 4}^{2}\right|}{\left(s_{\max 3}^{2}+s_{\max 4}^{2}\right)}$. Finally, $\Delta_{\text {circ }}=$ $2 d a$.

Acknowledgements. The authors warmly thank the Cluster scientific and technical teams, in particular the Cluster operation team in ESOC, as well as the Cluster science mission centre in JSOC, for making the tilt campaign come true. We are grateful to the FGM team and the Cluster Active Archive for providing magnetic field data and to the CDAWEB for providing environmental data. CNES and ESA funding supported WHISPER instrument construction and support WHISPER data analysis.

Topical Editor R. Nakamura thanks P.-A. Lindqvist and one anonymous referee for their help in evaluating this paper.

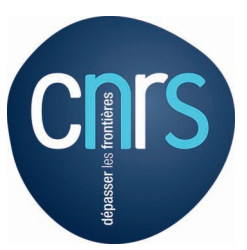

The publication of this article is financed by CNRS-INSU. 


\section{References}

Balogh, A., Dunlop, M. W., Cowley, S. W. H., Southwood, D. J., Thomlinson, J. G., Glassmeier, K. H., Musmann, G., Lühr, H., Buchert, S., Acuña, M. H., Fairfield, D. H., Slavin, J. A., Riedler, W., Schwingenschuh, K., Kivelson, M. G., and the Cluster magnetometer team: The Cluster magnetic field investigation, Space Sci. Rev., 79, 65-91, doi:10.1023/A:1004970907748, 1997.

Béghin, C., Décréau, P. M. E., Pickett, J., Sundkvist, D., and Lefebvre, B.: Modeling of Cluster's electric antennas in space: application to plasma diagnostics, Radio Sci., 40, RS6008, doi:10.1029/2005RS003264, 2005.

Boardsen, S. A., Eastman, T. E., Sotirelis, T., and Green, J. L.: An empirical model of the high-latitude magnetopause, J. Geophys. Res., 105, 23193-23219, doi:10.1029/1998JA000143, 2000.

Calvert, W.: Method for measuring the direction and wave mode of the radio echoes that are detected by a satellite radio sounder in the Earth's magnetosphere, Radio Sci., 34, 12871297, doi:10.1029/1999RS900005, 1999.

Carozzi, T., Karlsson, R., and Bergman, J.: Parameters characterizing electromagnetic wave polarization, Phys. Rev. E, 61, 2024 2028, 2000.

Carpenter, D. L. and Anderson, R. R.: An ISEE/Whistler model of equatorial electron density in the magnetosphere, J. Geophys. Res., 97, 1097-1108, doi:10.1029/91JA01548, 1992.

Cecconi, B.: Influence of an extended source on goniopolarimetry (or direction finding) with Cassini and Solar Terrestrial Relations Observatory radio receivers, Radio Sci., 42, RS2003, doi:10.1029/2006RS003458, 2007.

Cecconi, B. and Zarka, P.: Direction finding and antenna calibration through analytical inversion of radio measurements performed using a system of two or three electric dipole antennas on a three-axis stabilized spacecraft, Radio Sci., 40, RS3003, doi:10.1029/2004RS003070, 2005.

Cecconi, B., Lamy, L., Zarka, P., Prangé, R., Kurth, W. S., and Louarn, P.: Goniopolarimetric study of the revolution 29 perikrone using the Cassini Radio and Plasma Wave Science instrument high-frequency radio receiver, J. Geophys. Res., 114, A03215, doi:10.1029/2008JA013830, 2009.

Darrouzet, F., Décréau, P. M. E., De Keyser, J., Masson, A., Gallagher, D. L., Santolik, O., Sandel, B. R., Trotignon, J. G., Rauch, J. L., Le Guirriec, E., Canu, P., Sedgemore, F., André, M., and Lemaire, J. F.: Density structures inside the plasmasphere: Cluster observations, Ann. Geophys., 22, 2577-2585, doi:10.5194/angeo-22-2577-2004, 2004.

Darrouzet, F., Gallagher, D. L., André, N., Carpenter, D. L., Dandouras, I., Décréau, P. M. E., De Keyser, J., Denton, R. E., Foster, J. C., Goldstein, J., Moldwin, M. B., Reinisch, B. W., Sandel, B. R., and Tu, J.: Plasmaspheric Density Structures and Dynamics: Properties Observed by the CLUSTER and IMAGE Missions, Space Sci. Rev., 145, 55-106, doi:10.1007/s11214-008-9438-9, 2009.

Décréau, P. M. E., Fergeau, P., Krasnosel'skikh, V., Lévêque, M., Martin, P., Randriamboarison, O., Sené, F. X., Trotignon, J. G., Canu, P., Mögensen, P. B., and WHISPER Investigators: WHISPER, a resonance sounder and wave analyser: performances and perspectives for the Cluster mission, Space Sci. Rev., 79, 157193, doi:10.1023/A:1004931326404, 1997.
Décréau, P. M. E., Fergeau, P., Krasnoselskikh, V., Le Guirriec, E., Lévêque, M., Martin, Ph., Randriamboarison, O., Rauch, J. L., Senée, F. X., Séran, H. C., Trotignon, J. G., Canu, P., Cornilleau, N., de Féraudy, H., Alleyne, H., Yearby, K., Mögensen, P. B., Gustafsson, G., André, M., Gurnett, D. C., Darrouzet, F., Lemaire, J., Harvey, C. C., Travnicek, P., and Whisper experimenters: Early results from the Whisper instrument on Cluster: an overview, Ann. Geophys., 19, 1241-1258, doi:10.5194/angeo-19-1241-2001, 2001.

Décréau, P. M. E., Ducoin, C., Le Rouzic, G., Randriamboarison, O., Rauch, J.-L., Trotignon, J.-G., Vallières, X., Canu, P., Darrouzet, F., Gough, M. P., Buckley, A. M., and Carozzi, T. D.: Observation of continuum radiations from the Cluster fleet: first results from direction finding, Ann. Geophys., 22, 2607-2624, doi:10.5194/angeo-22-2607-2004, 2004.

Dušík, S., Granko, G., Safránková, J., Němeček, Z., and Jelínek, K.: IMF cone angle control of the magnetopause location: Statistical study, Geophys. Res. Lett., 37, L19103, doi:10.1029/2010GL044965, 2010.

Escoubet, C. P., Schmidt, R., and Goldstein, M. L.: CLUSTER - Science and mission overview, Space Sci. Rev., 79, 11-32, doi:10.1023/A:1004923124586, 1997.

Etcheto, J., Christiansen, P. J., Gough, M. P., and Trotignon, J.-G.: Terrestrial continuum radiation observations with GEOS-1 and ISEE-1, Geophys. Res. Lett., 9, 1239-1242, doi:10.1029/GL009i011p01239, 1982.

Fainberg, J., Hoang, S., and Manning, R.: Measurements of distributed polarized radio sources from spinning spacecraft: effect of a tilted axial antenna. ISEE-3 application and results, Astron. Astrophys., 153, 145-150, 1985.

Goldstein, J., Sandel, B. R., Frey, H. U., and Mende, S. B.: Multiple plasmapause undulations observed by the IMAGE satellite on 20 March 2001, J. Atmos. Sol.-Terr. Phys., 69, 322-333, doi:10.1016/j.jastp.2006.08.010, 2007.

Gough, M. P.: Non-thermal continuum emissions associated with electron injections: remote plasmapause sounding, Planet. Space Sci., 30, 657-668, doi:10.1016/0032-0633(82)90026-5, 1982.

Green, J. L., Sandel, B. R., Fung, S. F., Gallagher, D. L., and Reinisch, B. W.: On the origin of kilometric continuum, J. Geophys. Res., 107, 1105, doi:10.1029/2001JA000193, 2002.

Grimald, S., Décréau, P. M. E., Canu, P., Suraud, X., Vallières, X., Darrouzet, F., and Harvey, C. C.: A quantitative test of Jones NTC beaming theory using CLUSTER constellation, Ann. Geophys., 25, 823-831, doi:10.5194/angeo-25-823-2007, 2007.

Grimald, S., Décréau, P. M. E., Canu, P., Rochel, A., and Vallières, X.: Medium latitude sources of plasmaspheric non thermal continuum radiations observed close to harmonics of the electron gyrofrequency, J. Geophys. Res., 113, A11217, doi:10.1029/2008JA013290, 2008.

Grimald, S., Foullon, C., Décréau, P. M. E., Le Rouzic, G., Suraud, X., and Vallières, X.: Modulation of NTC frequencies by Pc5 pulsations : experimental test of the generation mechanism and magnetoseismology of the emitting surface, J. Geophys. Res., 114, A11211, doi:10.1029/2009JA014270, 2009.

Grimald, S., El-Lemdani Mazouz, F., Foullon, C., Décréau, P. M. E., Boardsen, S. A., and Vallières, X.: Study of non-thermal continuum patches: wave propagation and plasmapause study, J. Geophys. Res., 116, A07219, doi:10.1029/2011JA016476, 2011. 
Gurnett, D. A.: The Earth as a radio source: Terrestrial kilometric radiation, J. Geophys. Res., 79, 4227-4238, doi:10.1029/JA079i028p04227, 1974.

Gurnett, D. A.: The Earth as a radio source: The nonthermal continuum, J. Geophys. Res., 80, 2751-2763, doi:10.1029/JA080i019p02751, 1975.

Gurnett, D. A. and Shaw, R. R.: Electromagnetic radiation trapped in the magnetosphere above the plasma frequency, J. Geophys. Res., 78, 8136-8149, doi:10.1029/JA078i034p08136, 1973.

Gurnett, D. A., Calvert, W., Huff, R. L., Jones, D., and Siguira, M.: The polarization of escaping terrestrial continuum radiation, J. Geophys. Res., 93, 12817-12825, doi:10.1029/JA093iA11p12817, 1988.

Gurnett, D. A., Huff, R. L., and Kirchner, D. L.: The WideBand Plasma Wave Investigation, Space Sci. Rev., 79, 195-208, doi:10.1023/A:1004966823678, 1997.

Hanasz, J., Boudjada, M. J., Schreiber, R., Krawczyk, Z., Malycha, M., Mogilevsky, M. M., Rucker, H. O., and Romantsova, T. V.: Dynamic spectra of the Stokes parameters for the dayside and nightside Auroral Kilometric Radiation, Geophys. Res. Lett., 27, 1631-1634, doi:10.1029/1999GL010770, 2000.

Hoang, S., Fainberg, J., and Steinberg, J. L.: The $2 f_{\mathrm{p}}$ circumterrestrial radio radiation as seen from ISEE-3, J. Geophys. Res., 86, 4531-4536, doi:10.1029/JA086iA06p04531, 1981.

Huff, R. L., Calvert, W., Craven, J. D., Frank, L. A., and Gurnett, D. A.: Mapping of auroral kilometric radiation sources to the aurora, J. Geophys. Res., 93, 11445-11454, doi:10.1029/JA093iA10p11445, 1988.

Jones, D.: Terrestrial myriametric radiation from the earth's plasmapause, Planet. Space Sci., 30, 399-410, doi:10.1016/00320633(82)90046-0, 1982.

Jones, D.: A technique for studying density gradients and motions of plasmaspheric irregularities, J. Geophys., 52, 158-166, 1983.

Kasaba, Y., Matsumoto, H., Hashimoto, K., Anderson, R. R., Bougeret, J.-L., Kaiser, M. L., Wu, X. Y., and Nagano, I.: Remote sensing of the plasmapause during substorms: Geotail observation of nonthermal continuum enhancement, J. Geophys. Res., 103, 20389-20405, doi:10.1029/98JA00809, 1998.

Kurth, W. S., Baumback, M. M., and Gurnett, D. A.: Direction finding measurements of Auroral kilometric radiation, J. Geophys. Res., 80, 2764-2770, doi:10.1029/JA080i019p02764, 1975.

Kurth, W. S., Gurnett, D. A., and Anderson, R. R.: Escaping nonthermal continuum radiation, J. Geophys. Res., 86, 5519-5531, doi:10.1029/JA086iA07p05519, 1981.

Ladreiter, H. P., Zarka, P., Lecacheux, A., Macher, W., Rucker, H. O., Manning, R., Gurnett, D. A., and Kurth, W. S.: Analysis of electromagnetic wave direction finding performed by spaceborne antennas using singular-value decomposition techniques, Radio Sci., 30, 1699-1712, doi:10.1029/95RS02479, 1995.

Lamy, L., Zarka, P., Cecconi, B., and Prangé, R.: Auroral kilometric radiation diurnal, semidiurnal,and shorter term modulations disentangled by Cassini, J. Geophys. Res., 115, A09221, doi:10.1029/2010JA015434, 2010.

Lecacheux, A.: Direction finding of a radiosource of unknown polarization with short electric antennas on a spacecraft, Astron. Astrophys., 70, 701-706, 1978.
Lointier, G., Darrouzet, F., Décréau, P. M. E., Vallières, X., Kougblénou, S., Trotignon, J. G., and Rauch, J.-L.: Refilling process in the plasmasphere: a 3-D statistical characterization based on Cluster density observations, Ann. Geophys., 31, 217-237, doi:10.5194/angeo-31-217-2013, 2013.

Masson, A., Santolik, O., Carpenter, D. L., Darrouzet, F., Décréau, P. M. E., Green, J. L., Grimald, S., El-Lemdani Mazouz, F., Moldwin, M. B., and Nemec, F.: Advances in plasmaspheric wave research with CLUSTER and IMAGE observations, Space Sci. Rev., 145, 137-191, doi10.1007/s11214-009-9508-7, 2009.

Menvielle, M. and Berthelier, A.: The $K$-derived planetary indices: Description and availability, Rev. Geophys., 29, 415-432, doi:10.1029/91RG00994, 1991.

Morgan, D. D. and Gurnett, D. A.: The source location and beaming of terrestrial Continuum radiation, J. Geophys. Res., 96, 95959613, doi:10.1029/91JA00314, 1991.

Nagano, I., Wu, X.-Y., Takano, H., Yagitani, S., Matsumoto, H., Hashimoto, K., and Kasaba, Y.: Remote sensing the magnetosheath by the spin modulation of terrestrial continuum radiation, J. Geophys. Res., 108, 1224, doi:10.1029/2002JA009691, 2003.

Parrot, M., Santolík, O., Gurnett, D. A., Pickett, J. S., and Cornilleau-Wehrlin, N.: Characteristics of magnetospherically reflected chorus waves observed by CLUSTER, Ann. Geophys., 22, 2597-2606, doi:10.5194/angeo-22-2597-2004, 2004.

Pierrard, V. and Lemaire, J. F.: Development of shoulders and plumes in the frame of the interchange instability mechanism for plasmapause formation, Geophys. Res. Lett., 31, L05809, doi:10.1029/2003GL018919, 2004.

Reiner, M. J., Kaiser, M. L., Fainberg, J., Desh, M. D., and Stone, R. G.: $2 f_{\mathrm{p}}$ radio emission from the vicinity of the Earth's foreshock: WIND observations, Geophys. Res. Lett., 23, 1247-1250, doi:10.1029/96GL00841, 1996.

Shawhan, S. D. and Gurnett, D. A.: Polarization measurements of auroral kilometric radiation by Dynamics Explorer-1, Geophys. Res. Lett., 9, 913-916, doi:10.1029/GL009i009p00913, 1982.

Steinberg, J.-L., Hoang, S., and Lacombe, C.: Propagation of terrestrial kilometric radiation through the magnetosheath: ISEE-3 observations, Ann. Geophys., 7, 151-160, 1989.

Trotignon, J.-G., Décréau, P. M. E., Rauch, J. L., Vallières, X., Rochel, A., Kougblénou, S., Lointier, G., Facskó, G., Canu, P., Darrouzet, F., and Masson, A.: The whisper relaxation sounder and the Cluster active archive, in: The Cluster Active Archive, Studying the Earth's Space Plasma Environment, edited by: Laakso, H., Taylor, M. G. T. T., and Escoubet, C. P.: Astrophysics and Space Science Proceedings, 185-208, Springer, Berlin, doi:10.1007/978-90-481-3499-1_12, 2010.

Tsyganenko, N. A. and Stern, D. P.: Modeling the global magnetic field of the large-scale Birkeland current systems, J. Geophys. Res., 101, 27187-27198, doi:10.1029/96JA02735, 1996.

Woolliscroft, L. J. C., Alleyne, H. St. C., Dunford, C. M., Sumner, A., Thompson, J. A., Walker, S. N., Yearby, K. H., Buckley, A., Chapman, S., Gough, P., and the DWP investigators: The Digital Wave-Processing experiment on Cluster, Space Sci. Rev., 79, 209-231, doi:10.1023/A:1004914211866, 1997.

Zarka, P. and Kurth, W. S.: Radio wave emission from the outer planets before CASSINI, Space Sci. Rev., 116, 371-397, doi:10.1007/s11214-005-1962-2, 2005. 